\title{
Determining subpopulation methylation profiles from bisulfite sequencing data of heterogeneous samples using DXM
}

\author{
Jerry Fong1, Jacob R. Gardner², Jared M. Andrews ${ }^{3}$, Amanda F. Cashen ${ }^{4}$, \\ Jacqueline E. Payton ${ }^{\circledR 3}$, Kilian Q. Weinberger ${ }^{2}$ and John R. Edwards ${ }^{\circledR 1, *}$ \\ ${ }^{1}$ Center for Pharmacogenomics, Department of Medicine, Washington University School of Medicine, St. Louis, MO, \\ USA, ${ }^{2}$ Center for Data Science for Improved Decision Making, Department of Computer Science, Cornell University, \\ Ithaca, NY, USA, ${ }^{3}$ Department of Pathology and Immunology, Washington University School of Medicine, St. Louis, \\ MO, USA and ${ }^{4}$ Oncology Division, Department of Medicine, Washington University School of Medicine, St. Louis, \\ MO, USA
}

Received May 13, 2021; Editorial Decision June 01, 2021; Accepted June 04, 2021

\begin{abstract}
Epigenetic changes, such as aberrant DNA methylation, contribute to cancer clonal expansion and disease progression. However, identifying subpopulation-level changes in a heterogeneous sample remains challenging. Thus, we have developed a computational approach, DXM, to deconvolve the methylation profiles of major allelic subpopulations from the bisulfite sequencing data of a heterogeneous sample. DXM does not require prior knowledge of the number of subpopulations or types of cells to expect. We benchmark DXM's performance and demonstrate improvement over existing methods. We further experimentally validate DXM predicted allelic subpopulation-methylation profiles in four Diffuse Large B-Cell Lymphomas (DLBCLs). Lastly, as proof-of-concept, we apply DXM to a cohort of 31 DLBCLs and relate allelic subpopulation methylation profiles to relapse. We thus demonstrate that DXM can robustly find allelic subpopulation methylation profiles that may contribute to disease progression using bisulfite sequencing data of any heterogeneous sample.
\end{abstract}

\section{INTRODUCTION}

DNA methylation changes have been implicated in a variety of human diseases, including cancer (1). Methylation measurements are typically made from heterogenous samples consisting of multiple cell-types, which each have unique methylation patterns. As such, it is frequently difficult to interpret whether an observed change in methylation is due to a shift in sample composition or due to a true change in the methylation state of an underlying cell-type. For example, tumors are comprised of both normal cell types and cancer subclones. These subclones can acquire changes that increase their fitness, leading to faster cancer progression, treatment resistance and worse patient prognosis $(2,3)$. Though cancer subclones have generally been described with respect to genetic alterations, in principle epigenetic alterations such as DNA methylation could alter the expression of key genes in a subclone and impact its fitness. Moreover, in chronic lymphocytic leukemia (CLL), diffuse large B-cell lymphoma (DLBCL), acute myeloid leukemia (AML), Ewing Sarcoma, and glioblastoma, clonal heterogeneity in DNA methylation is associated with worse patient outcome (4-8). Unfortunately, even though subclonal methylation changes may be expected to underlie this observed heterogeneity, current methods do not effectively analyze subclonal methylation patterns from the bisulfite sequencing data of heterogeneous samples. One approach to address sample heterogeneity is fluorescence-activated cellsorting (FACS), but it is dependent on surface markers to distinguish cell types, which may not be known a priori or even exist for many cancer subclones. Hypothetically, single-cell bisulfite sequencing can address sample heterogeneity (9), but it is technically challenging and expensive. While this technology continues to develop, there remain hundreds of bisulfite sequencing datasets that have already been generated and are being generated that would remain useful to study. To understand the impact of methylation changes measured in these samples we thus need a computational approach to identify the number of underlying allelic subpopulations and their respective methylation profiles from the methylation data of a heterogeneous input sample.

\footnotetext{
*To whom correspondence should be addressed. Tel: +1 314362 6295; Fax: +1 314362 8844; Email: jredwards@wustl.edu Present address: Jacob R. Gardner, Computer and Information Science, University of Pennsylvania, PA, USA.

(C) The Author(s) 2021. Published by Oxford University Press on behalf of Nucleic Acids Research.

This is an Open Access article distributed under the terms of the Creative Commons Attribution License (http://creativecommons.org/licenses/by/4.0/), which permits unrestricted reuse, distribution, and reproduction in any medium, provided the original work is properly cited.
} 
Though extensive methods have been developed to describe subclonal architecture with respect to mutations or copy number variants (CNVs) (for a review see (10)), these methods cannot be easily adapted to methylation analysis since they typically assume subclonal events occur independently and are relatively rare. Unlike mutations or CNVs, methylation changes often occur in blocks of multiple $\mathrm{CpGs}$ over a short range called differentially methylated regions (DMRs) $(11,12)$. Additionally, there are many more aberrant changes in methylation than mutations or CNVs, with frequently $>100000$ DMRs observed in solid tumors as compared to at most $\sim 10000$ mutations or $\sim 100 \mathrm{CNVs}$ (13-15). Thus we need approaches that specifically model subclonal DNA methylation data.

Several analysis methods to determine the cellular composition and methylation profiles of subpopulation level events have been developed for epigenome-wide association studies (EWAS) (16-20). However, EWAS uses array-based technologies that probe the methylation state of $3 \%$ of the $\mathrm{CpGs}$ in the human genome, and as such, these approaches use assumptions and error-models that are not appropriate for sequence data. For example, they do not consider the strong local correlations of methylation changes, since adjacent probes are frequently $>1 \mathrm{~kb}$ apart (21). Consequently, there are not good options for those interested in deconvolving sequencing-based DNA methylation data such as obtained through WGBS, RRBS or capture methods.

Thus, we have developed DXM (Deconvolution of Subpopulations Existing in Methylation Data), a novel deconvolution strategy to identify the major allelic subpopulations and their respective methylation profiles from a heterogeneous sample (Figure 1A and B). DXM does not require explicit prior knowledge of the number of subpopulations or what types of cells to expect, and it provides a framework for considering methylation differences across multiple $\mathrm{CpGs}$ at the single-CpG resolution offered by bisulfite sequencing data. We benchmarked DXM on a wide set of simulated mixtures using bisulfite sequencing reads from sorted hematopoietic cell types and found that DXM outperformed methylPurify (22), another method developed for subclonal analysis of bisulfite sequencing data. We demonstrate that DXM can be used to study allele-specific methylation in the contexts of X-inactivation and imprinting. We further conducted Agilent Methyl-Seq bisulfite sequencing analysis in four samples from patients with DLBCL, a B-cell lymphoma derived from germinal center B cells. We validated that DXM predictions for subpopulation methylation profiles were recapitulated in relevant sorted $\mathrm{CD} 4^{+} \mathrm{T}$ and $\mathrm{CD} 19^{+} \mathrm{B}$ cells from these samples. As proofof-concept, we applied DXM to bisulfite sequencing data from a cohort of 31 DLBCL samples (5) to highlight how DXM can be used to analyze subpopulation methylation in heterogeneous cancer samples and relate them to relapse.

\section{MATERIALS AND METHODS}

\section{Public datasets}

Bisulfite sequencing data was downloaded from the Roadmap Epigenomics project (23) (REP), the Blueprint Epigenome Project (24) (BEP), ENCODE (25) and GEO (Accession: GSE29069 (26), GSE66329 (27), GSE75868 (for the WIBR3 cell-lines) (28). A full list is provided in Supplementary File 1 (Supplementary_File_1.xlsx).

\section{DXM}

Using sequencing-based DNA methylation data from heterogeneous samples as input, DXM deconvolves data across a set of user-defined regions and outputs the number of allelic subpopulations as well as the methylation profile for each subpopulation across each region (Figure 1A and B). We adopt an iterative scheme to solve for the number of subpopulations, beginning with only 1 major subpopulation, and adding additional subpopulations provided that they do not cause an overfit. This approach does not assume how many underlying subpopulations are present a priori, but it takes an Occam's Razor approach in determining the minimal number of subpopulations that can reasonably explain the observed data.

DXM input. DXM uses processed data as input, consisting of a BED-like format containing tab-delimited columns of chromosome names, start position, end position, methylation level (mCG/CG), sequencing coverage, and region id. DXM performs deconvolution over user-specified intervals (e.g. promoter regions, CGIs, or enhancers) and is compatible with any reference genome. While input data does not need to be filtered for a specific coverage cutoff, we recommend a coverage of at least 4 reads per $\mathrm{CpG}$ for more reliable results. Typically, we recommend collapsing $\mathrm{CpG}$ data across strands to increase reliability of the methylation estimates, but this is not required. Additional pre-processing details for specific experiments are detailed in their respective sections below.

Minimization. We next estimate the best possible prevalence of subpopulations from the distribution of all fractional methylation values detected. We minimize the L1-difference between the expected underlying fractional methylation values and the original methylation distribution. The expected underlying fractional methylation values represent a set of all possible combinations for detected methylation given that each subpopulation will be methylated or not. For example, if there are two subpopulations with prevalence of 0.3 or 0.7 in the sample, then the expected fractional methylation values detected are $\{0,0.3,0.7,1\}$. In the (unlikely) event of a tie, the solution with the smallest possible subpopulation is selected. After minimization, each region is solved independently for its methylation profiles.

Modified hidden markov model. Given the number of underlying subpopulations and their expected prevalence, we solve for the most likely methylation profiles by applying the Viterbi algorithm to a modified Hidden Markov Model (HMM). An HMM is well-suited to model sequence data with local correlations, as exhibited by DNA methylation (Supplementary Figure S1a-d) (29). In brief, the HMM in DXM first considers how likely a given methylation profile would contribute to the observed bisulfite sequencing data. It then extends this logic for how likely a mixture of these methylation profiles would contribute to the observed data. The underlying methylation sequence of the CpGs 

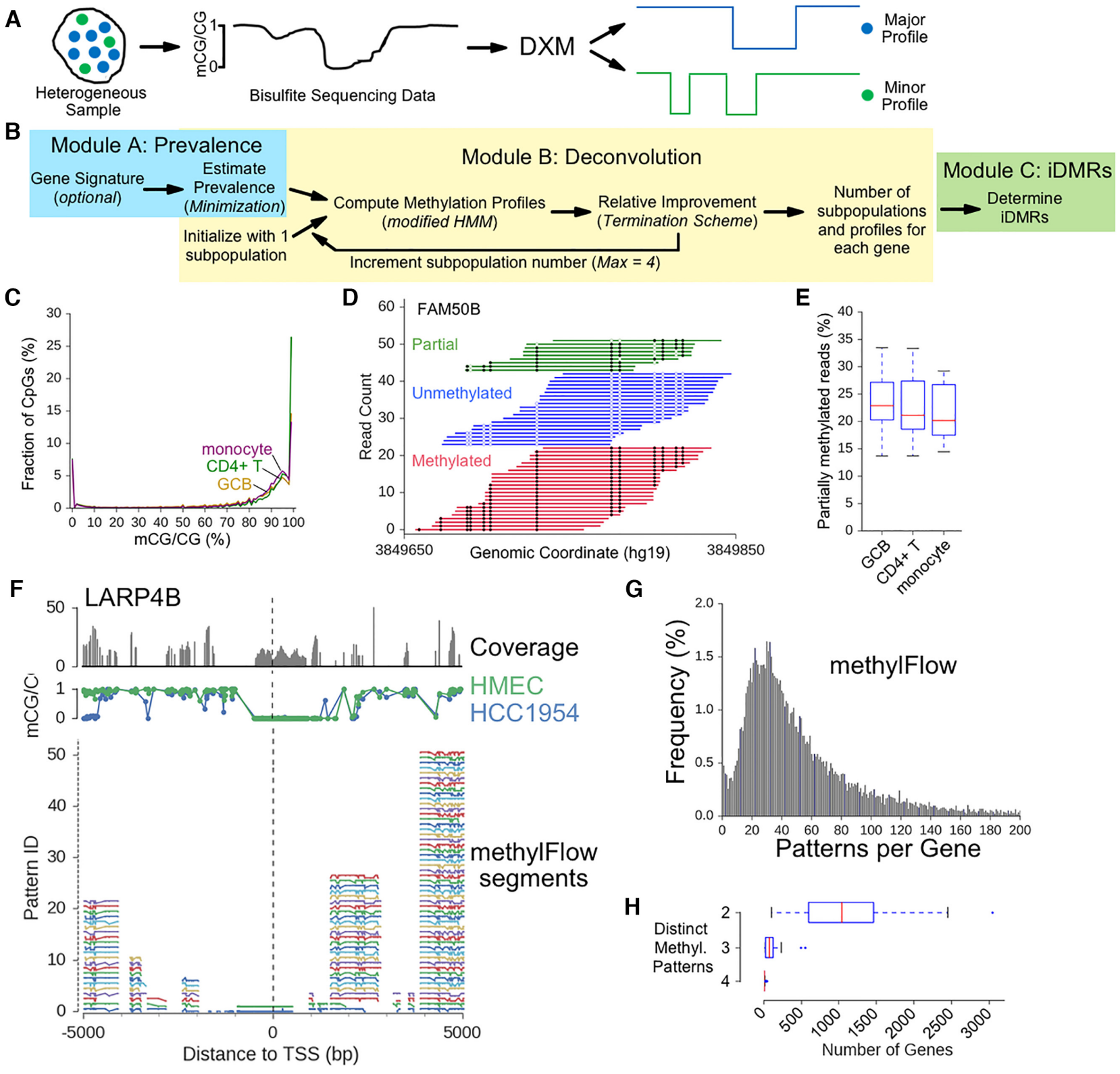

E
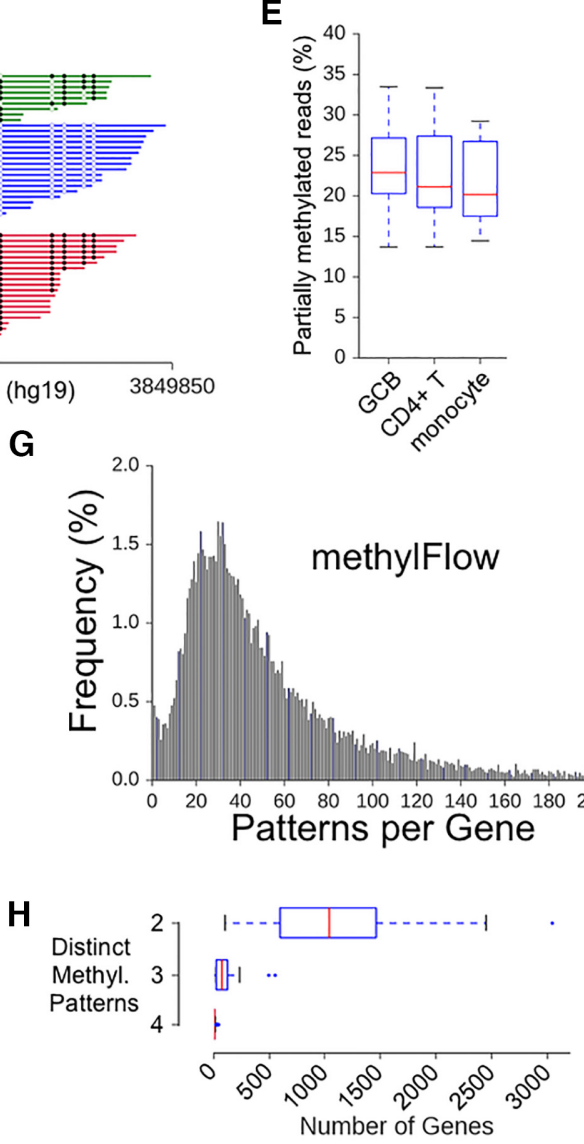

Figure 1. DXM scheme and design considerations. (A) DXM takes bisulfite sequencing data from a heterogeneous sample and identifies its underlying allelic subpopulation methylation profiles and the number of subpopulations. (B) DXM consists of three modules: Module A estimates the prevalence, Module B solves subpopulation methylation profiles, and Module C calls intrasample DMRs (i-DMRs). (C) Fractional methylation distribution for sorted cell types. $12.4 \%, 15.5 \%$ and $22.7 \%$ of CpGs are partially methylated (i.e. the fraction of CpGs between $20-80 \%$ methylation) in CD4+ T-cells (green), GCB cells (gold), and monocytes (purple), respectively. (D) Bisulfite sequencing reads across part of the imprinting control region for FAM50B in germinal center B-cells (GCBs) from BEP. Open circles are unmethylated CpGs and closed circles are methylated. (E) The percentage of partially methylated reads at 19 well-characterized imprinted loci in different sorted cell types from BEP. (F) methylFlow (MF) outputs for LARP4B from analysis of a $22 \times$ coverage HMEC-HCC1954 mixture (35:65). For individual segments, MF outputs between 1 and 52 potential profiles. (G) The most profiles in any individual segment by MF for all promoters in a $22 \times$ coverage HMEC-HCC1954 mixture (35:65). (H) Number of gene promoters with DMRs identified between or among different sorted cell types from BEP (CD4T, CD8T, erythroblast, eosinophil, hematopoietic multiprogenitor, GCB, megakaryocyte, monocyte, osteoclast). Promoters were considered to have distinct methylation patterns if there was a DMR identified between each pair of cells considered (e.g. for cell types $\mathrm{A}, \mathrm{B}$ and $\mathrm{C}$, there are three distinct patterns if there is a DMR between $\mathrm{A}-\mathrm{B}, \mathrm{B}-\mathrm{C}$ and $\mathrm{A}-\mathrm{C}) .17450$ total genes were considered. 
of all profiles represents the state-sequence. The transition probabilities are the likelihood that a cell type would have a certain underlying methylation sequence for its $\mathrm{CpGs}$, given the probability that two nearby $\mathrm{CpG}$ sites have the same methylation state as a function of the distance between them. The 1 are the likelihood of a CpG's underlying methylation state across all subpopulations contributing to the observed bisulfite sequencing data as modeled by a beta-binomial distribution. This setup allows the HMM to holistically consider methylation data from all $\mathrm{CpGs}$ in the region and account for data quality of each $\mathrm{CpG}$ (coverage). The HMM states, transitions, and emissions are described in greater detail below.

HMM states. For our modified HMM, if there are $d$ subpopulations to consider, a state is a length- $d$ vector of the binary methylation values of each underlying subpopulation at a given $\mathrm{CpG}$.

HMM transition probabilities. The transition probability represents the change in methylation of each underlying subpopulation from one $\mathrm{CpG}$ to an adjacent $\mathrm{CpG}$. To calculate the transition probability, we have defined a set of $2 \times$ 2 transition matrices that capture transitions of one subpopulation's methylation state from one $\mathrm{CpG}$ to the next. From this set, the transition matrix is selected based on the distance between $\mathrm{CpGs}$, which captures correlations in methylation and distance seen in Supplementary Figure S1a-d. We consider each subpopulation's transition to be independent and identically distributed, so the transition probability is ultimately calculated from $d$ identical transition matrices.

To ensure robustness, transition probabilities were trained on somatic tissue and cell-line datasets available via REP, and all testing was conducted on datasets of different cell types available for BEP (Supplementary_File_1.xlsx) or GSE29069. Briefly, for all CpGs in a dataset, the detected fractional methylation was rounded to the closest binary methylation state (fully unmethylated or fully methylated). Transitions between binary methylation states of adjacent $\mathrm{CpGs}$ were then counted (empirical sampling). We elected to group all transitions greater than $1000 \mathrm{bp}$ in the same category when selecting transition probabilities to use. Notably, this training includes correlations across genomic elements, including open-sea regions and $\mathrm{CpG}$ islands, as we did not observe a large difference in correlations across genic or intergenic regions (Supplementary Figure S1b-d). We do not utilize any cell type-specific transition probabilities for underlying subpopulations. Importantly, we observed similar correlations across multiple datasets, including different assays for methylation sequencing (WGBS, RRBS, Agilent Methyl-Seq), suggesting DXM can be applied to any genome-wide or reduced representation methylation sequencing dataset.

$$
\begin{aligned}
P(b, n)= & \sum_{i=0}^{n} \sum_{j=0}^{\min (i, b)}\left(\begin{array}{l}
n \\
i
\end{array}\right) p^{i}(1-p)^{n-i} \\
& * \frac{\left(\begin{array}{c}
i \\
j
\end{array}\right)\left(\begin{array}{l}
n-i \\
b-j
\end{array}\right)}{\left(\begin{array}{l}
n \\
b
\end{array}\right)} * m(i, j) * u(n-i, b-j)
\end{aligned}
$$

Emission probabilities. The emission probabilities, $P(b, n)$, at each $\mathrm{CpG}$ site were calculated from Equation (1), which has terms to capture the prevalence of each subpopulation, the binomial sampling error, and measurement errors. In this equation, $n$ is the total number of reads (coverage) at that $\mathrm{CpG}$ site, $b$ is the number of methylated reads, $i$ is the number of reads that came from the underlying methylated state, $m(i, j)$ is the probability that $j$ of these $i$ reads are correctly measured as methylated reads, $u(n-i, b-j)$ is the probability that $b-j$ of the $n-\mathrm{i}$ unmethylated reads are incorrectly measured from the unmethylated state (i.e. measured as methylated), and $p$ is the prevalence of all subpopulations with an underlying methylated state. Assuming reads are independently and identically distributed among allelic subpopulations as a function of prevalence, reads can be grouped by whether they came from an allelic subpopulation whose underlying state was methylated or not. Thus, if $i$ reads came from subpopulations with an underlying state of methylated, $n-i$ come from those with an underlying state as unmethylated, and this case follows a binomial distribution, where $p$ is the prevalence of all subpopulations with an underlying methylated state. Of these $i$ reads, $j$ may be observed as methylated, and assuming all reads are independent, the probability of this event follows a hypergeometric distribution. Lastly, the probability that $j$ of $i$ reads are observed as methylated, given that the expected underlying state is fully methylated, is modeled with a beta-binomial distribution $m(i, j)$. A separate beta-binomial distribution, $u(n-i, b-j)$, models a similar probability that $b-j$ of $n-$ $i$ reads are observed as methylated given an expected underlying state of fully unmethylated. Determination of the beta-priors for these beta-binomial distributions is provided below (see below in Beta-binomial fit). Summing across all valid combinations of $i, j$ gives the final emission probabilities. We use log-probabilities in all calculations to ensure precision.

Termination scheme. DXM determines the minimum number of subpopulations that explain the data by terminating when adding another allelic subpopulation no longer improves the fit to the data. Let $p(x)$ represent the probability that the state-sequence $x$ correctly explains the data and let $x_{r}$ represent the most likely statesequence for $r$ subpopulations. DXM will consider an additional subpopulation to be valid if there is an increase in likelihood, that is if $p\left(x=x_{r+1}\right)>p\left(x=x_{r}\right)$. By Bayes' rule, this can be written as $p\left(x=x_{r+1} \mid r+1\right) * p(r+1)>$ $p\left(x=x_{r} \mid r\right) * p(r)$, where $p(r)$ is the probability that $r$ subpopulations best explain the underlying data. In the uninformed case, we would have a uniform prior on the number of subpopulations to expect, e.g. $p(r)=p(r+1)$, and thus, the comparison is $p\left(x=x_{r+1} \mid r+1\right)>p\left(x=x_{r} \mid r\right)$. The conditional probability of a state-sequence $x_{r}$ explaining the data given $r$ subpopulations, $p\left(x=x_{r} \mid r\right)$, is solved by the Viterbi algorithm, so termination is achieved when the relative probability of a solution is worse. Though it is possible that our scheme does not yield the global solution $\left\{\right.$ e.g. $p\left(x=x_{r+1} \mid r+1\right) \leq p\left(x=x_{r} \mid r\right)$ but $\left.p\left(x=x_{r+2} \mid r+2\right)>p\left(x=x_{r} \mid r\right)\right\}$, we have not observed this in practice and believe the case to be highly unlikely for reasonably well-behaved beta-binomial distributions. Additionally, this scheme is guaranteed to converge. 
Consider the case where an additional subpopulation has the same methylation profile as an existing one. In this case, the emission probability would remain unchanged, but the transition probabilities would be less likely since there is an additional subpopulation to consider. Since there are a finite number of possible methylation profiles for $\mathrm{g} \mathrm{CpGs}$ $\left(2^{\mathrm{g}}\right)$, this case is guaranteed, though in practice we need to consider far fewer than $2^{g}$ methylation profiles (e.g. four total profiles).

DXM output. The output of DXM includes an estimate to the number of underlying subpopulations, their respective methylation profiles across each user-defined region in BED-like format, and an estimate of their relative prevalence in the sample. Given the utility of identifying differentially methylated regions (DMRs), we have also provided a post-hoc utility to identify intrasample DMRs between or among identified subpopulation methylation profiles (i-DMRs). Throughout this work we require i-DMRs to be least 50bp in length, contain at least three CpGs, and have at least $90 \%$ of the CpGs are differentially methylated. These are similar to the filtering parameters used by the DMR-caller DSS (30). DXM i-DMRs can be ranked based on their improvement in relative probability when modeled with two subpopulations as compared to 1 , given as $p\left(x=x_{2} \mid 2\right)-p\left(x=x_{1} \mid 1\right)$, which provides compatibility with DXM results for analyses such as gene set enrichment.

\section{Beta-Binomial fit}

An empirical beta-prior for use in DXM was drawn from training data from REP. This reflects an expectation of how fractional methylation of a given $\mathrm{CpG}$ is detected across all reads. In a bisulfite sequencing experiment, we consider that every read represents an independent sampling of the methylation value of the given $\mathrm{CpG}$. For an expected unmethylated state, every $\mathrm{CpG}$ with percent methylation between $0 \%$ and $40 \%$ was considered; similarly, $60-100 \%$ were the cutoffs for an expected methylated state. The methylation of each $\mathrm{CpG}$ was rounded to the nearest $0.5 \%$, and these discrete empirical distributions were then normalized. To find a suitable beta prior for this distribution, the probability density function of a given beta distribution was evaluated at each domain value in the empirical distribution (e.g. every $0.5 \%$ methylation). This new distribution was then normalized, and the L1-difference between it and the empirical distribution was minimized. All alpha/beta parameters for underlying beta distributions were fixed as integers, and a linear combination of beta distributions was employed to better capture spiking behavior near the edges of the distribution (e.g. $0 \%$ or $100 \%$ methylation) and a small spike around $50 \%$ methylation (due to imprinting and allele-specific methylation).

\section{Region definitions}

Gene promoter windows. Genes were defined using RefSeq (31) annotations, where only genes (transcripts) with 'cmpl' annotations for both the coding start and end sites were used. For genes with multiple transcription start sites
(TSS), only the first was considered. Only genes on autosomal chromosomes were considered for analysis. Gene promoter windows were then defined as the region $\pm 5 \mathrm{~kb}$ from the annotated TSS. While there is no agreed upon definition of a gene promoter, this region was chosen because it is larger than available definitions and should therefore include the entire promoter for analysis. Additionally, this window has previously been shown to be useful for predicting expression changes from DNA methylation (32).

Imprinted regions. Imprinted regions were taken from a list of known imprinted regions (Table 1 in Court et al. 2014) (33).

$X$-inactivated $C p G$ islands. $\quad \mathrm{CpG}$ islands on chromosome $\mathrm{X}$ were defined by UCSC annotations. These were then filtered for association with genes as done for gene promoter windows (refGene.txt file from UCSC with a 'cmpl' annotation for coding start and end sites.).

Enhancers. For each cell-type, we obtained H3K27Ac and H3Kmel ChIP-Seq data from BEP (GCB, EGAD00001002442; Monocyte, EGAD00001002523). Active enhancers were defined as regions with overlapping $\mathrm{H} 3 \mathrm{~K} 27 \mathrm{Ac}$ and $\mathrm{H} 3 \mathrm{Kmel}$ peaks (using bedtools intersect). Enhancers were further annotated as cell-type specific or common for pairs of cell-types based on whether they overlapped (bedtools intersect, using an outer join to consider as wide an overlap as possible). To remove any regions that overlapped promoters, we used bedtools to remove any enhancers that overlapped the region $\pm 1 \mathrm{~kb}$ around any TSS (refSeq, genes with a 'cmpl' CDS start and end annotation). Finally, only autosomal enhancers with methylation data at $\geq 4 \mathrm{CpGs}$ with $\geq 20$ reads were used for analysis.

\section{Methylation-level mixture simulations}

First, reference cell-type profiles for HMEC (Human mammary epithelial cells), CD4+ T and HCC1954 breast cancer cells were generated by taking the average methylation level (mCG/CG) for each $\mathrm{CpG}$ from published data (see Supplementary_File_1.xlsx for accession numbers) and rounding to 0 or 1 to binarize the profile for each individual sample. $\mathrm{CpGs}$ were only considered if there were at least 4 reads. For each simulation at a fixed prevalence and a fixed coverage, 1000 gene promoter regions ( $\pm 5 \mathrm{~kb}$ from the TSS) were randomly selected. The mixed methylation level (mCG/CG) of each $\mathrm{CpG}$ was then computed as the dot product of the prevalences and methylation values. Binomial sampling was then used to simulate the measured methylation value of the mixed sample based on the chosen coverage. DXM was then used to deconvolve the mixture.

\section{Read-level mixture simulations}

For simulated mixture generation, bisulfite sequencing reads (from BEP or GSE29069 datasets) were mapped with bsmap2.90 (34) against the hg19 genome using the -R flag. Mapped bisulfite sequencing reads were coordinate-sorted with tabix (35), after which all reads overlapping the TSS \pm 
$10 \mathrm{~kb}$ window of genes were taken for input (a larger window was used to ensure that all promoter overlapping reads were included). Next, reads were subsampled with two parameters: the expected average coverage of the total mixture, and the expected prevalence of each underlying cell type. Given an expected average coverage, the expected total number of reads was calculated as (coverage * domain size / read length). Average read length was $100 \mathrm{bp}$, and domain size was estimated as the number of genes $\times 10 \mathrm{~kb}$ (the size of the TSS $\pm 5 \mathrm{~kb}$ window). Next, the expected number of reads from each cell type was calculated as prevalence $\times$ total reads. Given the number of reads from each cell type, we calculated a sampling rate for each file, assuming singleend reads are present. This setup allows us to sample differences in coverage across each region and does not impose that more reads must come from the more prevalent cell type, which may not be true upon sequencing. Once a simulated mixture was generated, its processed methylation was obtained using the methRatio.py script provided with bsmap, and methylation data from both strands of DNA were consolidated for analysis. CpGs with fewer than 4 reads or more than 1000 reads were excluded from analysis. Similarly, read-level mixtures for enhancers were generated using the coordinates for the enhancer region instead of the window around the TSS.

\section{DMR calls}

We utilized DSS (30) as an independent DMR-caller to help evaluate DXM results. DSS uses a Bayesian hierarchical model and was run using the following parameters: equal dispersion $=$ True (for comparing single samples against each other), deltaVal $=0.3$ ('30\% methylation difference'), and a pval threshold of 0.05 (a $\mathrm{CpG}$ is considered differentially methylated if this threshold is met).

\section{Identifying regions with distinct methylation signatures in mixtures of more than two cell types}

Promoter regions $( \pm 5 \mathrm{~kb}$ around the TSS) were considered to have distinct methylation patterns if there was a DMR identified between each pair of cells considered. For example, for cell types A, B and C, a region is considered to have three distinct patterns if there is a DMR between $\mathrm{A}-\mathrm{B}, \mathrm{B}-\mathrm{C}$ and $\mathrm{A}-\mathrm{C}$ in the region.

\section{Region signatures for prevalence calling}

Region signatures for prevalence calling were defined by first running DSS to identify DMRs between germinal center B cells (GCB, Blueprint: T14_11) and monocytes (Blueprint: S000RD13). Next, this list was further filtered for genes that showed differential expression (FPKM $>5$ per sample, $>$ two log-fold difference) between the two samples (Blueprint T14_11 and S000RD).

\section{MethylPurify and methylflow}

MethylPurify (22) and methylFlow (36) were run with default settings except as noted below. For methylPurify, the most informative bins were identified from the '* . Informative_bins.bed.OneForCGI' file, and subpopulation profiles were found in the MethylProfile.bed file. For the mixture of HMEC and HCC1954 cells methylPurify was run with default parameters. For GCB and monocyte mixtures, both CGI and $\pm 5 \mathrm{~kb}$ around the TSS of all RefSeq genes were used as windows for the analysis, and the methylPurify code was altered to not filter reads $\geq 100 \mathrm{bp}$. For methylFlow, the methylation profiles of individual segments were obtained from the 'patterns.tsv' file, where pattern id (pid) refers to the number of profiles solved for a segment.

\section{Partial methylation in imprinted regions}

All reads overlapping the region for at least $1 \mathrm{bp}$ were found using tabix (part of samtools). For reads overlapping the region boundary, only the part of the read that overlapped with the imprinted region were considered to determine its partial methylation status. If all $\mathrm{CpGs}$ covered by a read in the imprinted region were fully methylated or fully unmethylated, the read was considered to be non-partially methylated; otherwise it was labelled as partially methylated. Reads are equivalent to fragments since data are from single-end reads.

\section{Evaluation metrics}

To evaluate whether DXM-solved methylation profiles resembled those of reference cell-types, we first computed the 'closest possible' reference methylation profile by rounding all fractional methylation values of the reference cell types to either 0 (fully unmethylated) or 1 (fully methylated). DXM-solved methylation profiles were then compared against each closest possible reference methylation profile and assigned to the reference that differed at the fewest number of CpGs (e.g. L1-norm difference). During assignment, DXM-solved subpopulations were not forced to be associated to different reference cell types, and assignment was allowed even in cases where there was significant discrepancy (e.g. $>50 \%$ of CpGs).

Accuracy was reported as the percent of $\mathrm{CpGs}$ that were incorrect out of all $\mathrm{CpGs}$ for that gene promoter. After each profile was assigned to a reference cell type, to evaluate how accurate DXM was in identifying cell types at the correct relative prevalence, the major allelic subpopulation was defined from the expected prevalence of the underlying subpopulations in the mixture. The percent of major subpopulation profiles DXM identified that corresponded to the correct expected major cell type was then computed.

\section{Ontology analysis}

Ontology analysis was conducted using the DAVID functional annotation tool with default parameters and background as $H$. sapiens.

\section{Primary samples}

Primary DLBCL samples from lymph node biopsies were obtained from the WUSM Lymphoma Banking Program. Written informed consent was obtained from all patients as part of the WUSM Lymphoma Banking Program. This study was approved by the Washington University in St. 
Louis Institutional Review Board (\#201710120). A full table of sample characteristics is provided in (Supplementary Table S1). Clinical flow cytometry was performed by the Barnes-Jewish Hospital clinical laboratory at the time of biopsy using standard clinical protocols for CD3+ $\mathrm{T}$ and CD19+ B cells. Sections of biopsy for tissue banking were processed to single cell suspensions and cryopreserved at $>10$ million cells/ml by the WUSM Tissue Procurement Facility. Samples were flash-thawed at $37^{\circ} \mathrm{C}$ and pelleted at 200 $\mathrm{rpm}, 5 \mathrm{~min}, 4^{\circ} \mathrm{C}$. Samples were then resuspended in wash buffer (4\% Fetal Calf Serum in dPBS). Half of each sample was isolated for Agilent-Methyl Seq, and the remaining half of each sample was sent for cell sorting.

\section{Cell sorting (FACS)}

Each sample was first blocked with 100ul of 5\% Human TruStain FcX (Biolegend) in wash buffer (4\% FCS in dPBS) for $7 \mathrm{~min}$ at room temperature. Samples were pelleted $(200 \mathrm{~g}$, $5 \mathrm{~min}, 4^{\circ} \mathrm{C}$ ) and resuspended in $100 \mathrm{ul}$ of the following binding buffer: 20ul (1 test volume) mouse anti-human CD19PE (BD Biosciences), 20 ul (1 test volume) mouse antihuman CD4-FITC (BD Biosciences), 5ul (1 test volume) 7AAD (BD Biosciences), and $45 \mathrm{ul}$ wash buffer. Samples were incubated in the dark at $4^{\circ} \mathrm{C}$ for $20 \mathrm{~min}$. Following incubation, samples were washed twice with wash buffer before sorting with BD FACSAriaII at a standard flow rate for PE, FITC, and PerCP-Cy5.5-A channels. Standard FSC and SSC gates were used to identify lymphocytes. CD4+/CD19/7-AAD- cells and CD19+/CD4-/7-AAD- cells were collected for subsequent analysis.

\section{Agilent methyl-Seq analysis}

Genomic DNA was isolated from each sample using the Zymo Quick-DNA Miniprep kit. Agilent SureSelect Methyl-Seq was then conducted for each sample following manufacturer's recommendations for 1ug of input gDNA. Briefly, gDNA was sheared using a Covaris instrument at the recommended settings to an expected fragment size 150-200 bp, as confirmed by $2 \%$ agarose gel. Samples underwent end-repair, poly-A tailing, and ligation of methylated adapters to facilitate capture enrichment. DNA was resuspended in nuclease-free water as recommended and underwent vacuum-concentration to a volume of $<5 \mathrm{ul}$. This methylated-adapter ligated DNA was then allowed to hybridize to the capture regions as per manufacturer recommendations. Next, bisulfite conversion was conducted on the input DNA at $64^{\circ} \mathrm{C}$ for $2.5 \mathrm{~h}$ using the EZ DNA Methylation-Gold kit (Zymo), After conversion, libraries were PCR amplified and then indexed using provided indices in the Agilent Methyl-Seq kit. Samples were sequenced with an Illumina Hi-Seq3000 $(2 \times 150$ pairedend reads). Sequencing specifications are found in a table. On average, we obtained $>50 \times$ coverage across $\sim 4.9$ million $\mathrm{CpGs}$ for each patient, and we observed a minimum bisulfite conversion efficiency of $>98.9 \%$ for all samples, as estimated by nonCpG conversion rate. All data were then aligned with biscuit v0.3.8 (https://huishenlab.github. io/biscuit/) and processed methylation data were obtained with the BISCUIT pipeline as recommended in the quick start page (pileup and vcf2bed commands). Data was collapsed per strand, and only $\mathrm{CpG}$ methylation data was considered.

\section{Targeted bisulfite sequencing}

A table of all bisulfite sequencing primers and locations is provided in Supplementary Table S2. Bisulfite primers for loci of interest were designed using methPrimer2.0 (37) with default settings and BiSearch (38), requiring only one major genomic location for expected amplification. Primers were temperature-optimized for PCR amplification (QIAGEN Pyromark PCR kit) using bisulfite converted gDNA (as above) from GM12878 cells. gDNA was isolated and bisulfite converted for each sample as above. PCRamplification was conducted for each region in each sample using 100pg of bisulfite-converted DNA as input. PCR products were barcoded and made into Illumina sequencing libraries as in (39). Libraries were sequenced on an Illumina Hiseq 4000. Sequences were trimmed for adaptors and poor-quality sequence using TrimGalore (https://github. com/FelixKrueger/TrimGalore) with default parameters. Sequences were then mapped to their target regions using Bismark (40) and default parameters and methylation levels (mCG/CG) extracted with bismark_methylation_extractor using the appropriate flag to collapse methylation across strands and otherwise default parameters.

\section{Enrichment analysis}

To determine the enrichment of CpGs associated with iDMRs in genomic elements $(\mathrm{CpG}$ island, shore, other elements), the observed distribution of CpGs in i-DMRs for these elements was compared to the expected distribution of CpGs in the TSS $\pm 5 \mathrm{~kb}$ window that were detected by Agilent Methyl-Seq for these genomic elements. To determine the enrichment of iDMRs in the region around the TSS, we plot the $\log _{2}$-ratio of the observed normalized $\mathrm{CpG}$ counts in i-DMRs to the expected normalized $\mathrm{CpG}$ counts of CpGs for a given position relative to the TSS seen in either Agilent Methyl-Seq or WGBS.

\section{Statistics}

Distributions were compared using Student's t-test for two groups or ANOVA with Tukey's posthoc $t$-test for multiple groups. Cohen's $d$-test was used to estimate effect size. Chi-square test for proportions was used to compare distributions of $\mathrm{CpGs}$ for enrichment analysis.

\section{RESULTS}

\section{Features of methylation distributions in sorted cells}

Before developing a deconvolution strategy, we sought to understand what constitutes distinct DNA methylation profiles in bisulfite-sequencing data both globally and in imprinted regions. We first examined the distribution of methylation levels (mCG/CG) within individual sorted cell types from the Blueprint Epigenome Project (BEP). One would expect that within a sorted cell type there would be 
relatively sharp peaks at $0 \%, 50 \%$ and $100 \%$ corresponding to unmethylated, imprinted or allele-specifically methylated, and methylated CpGs. However, from inspecting the distribution of methylation values, there are a variety of intermediate methylation values (i.e. not fully methylated or unmethylated) as well as substantial shoulders to the expected peaks around $0 \%$ and 100\% methylation (Figure 1C). We next examined methylation in three types of blood leukocytes at imprinted loci. Imprinted loci are characterized by a DMR that is fully methylated in one inherited allele and fully unmethylated in the other $(33,41)$. Despite this, we observe a substantial number of partially methylated reads as seen for the DMR near FAM50B in Figure 1D (other examples are in Supplementary Figure S2a). In fact, we find that on average $26.6 \%$ of bisulfite sequencing reads across DMRs in three hematological cells show partial methylation, or evidence for methylated and unmethylated CpGs on the same allele (Figure 1D, E, Supplementary Figure $\mathrm{S} 2 \mathrm{a})$. This results in a fractional methylation distribution at imprinted DMRs that is wider than what might be expected based on binomial sampling for a profile with $50 \%$ methylation (one methylated and one unmethylated allele, Supplementary Figure S2b).

These deviations from idealized distributions at imprinted domains, and fully methylated and unmethylated $\mathrm{CpG}$ sites likely do not arise from technical issues since both sequencing read quality (Supplementary Figure S2c) and bisulfite conversion efficiency are high $(>99.7 \%$ overall reported for REP, (42), Supplementary Figure S2d). Moreover, local variation in bisulfite conversion efficiency in these regions does not drive the observed methylation variability seen in partially methylated reads (Supplementary Figure S2d, e). As such, these observations likely represent noise due to further biological heterogeneity (e.g. more than two underlying states) or biological noise such as ageassociated drift (43) that is frequently smoothed to facilitate interpretation. In addition, the fractional methylation of a $\mathrm{CpG}$ across all reads appears to be insufficiently modeled by a binomial distribution defined by the number of reads and expected average methylation to model the counting errors due to sampling. Thus, as we developed our deconvolution strategy, we incorporated a beta-binomial distribution to model additional observed errors in the fractional methylation of each $\mathrm{CpG}$ across reads. The parameters of the beta-binomial are learned from real-sequencing data.

\section{Smoothing individual methylation profiles leads to a solution that is easier to interpret}

We next applied methylFlow (MF) (36) to a simulated mixture of reads from whole-genome bisulfite sequencing of HMEC (Human Mammary Epithelial Cells) and HCC1954 (breast tumor) cells to understand whether interpreting every methylation change at each $\mathrm{CpG}$ as an individual pattern could lead to a useful result. methylFlow uses a network flow analysis to stitch together segments of methylation patterns and then combine these segments to identify underlying methylation profiles supported by bisulfite sequencing reads from an experiment. We focused on a wide window around the gene's transcription start site (TSS $\pm 5 \mathrm{~kb})$ that is a conservative estimate for a region that likely includes the gene promoter and where methylation differences are most predictive of gene expression (44). From a $22 \times$ coverage simulated mixture of HMEC:HCC1954 (35:65) methylFlow identified 99.5\% (17 367 of 17450 ) gene promoters (TSS $\pm 5 \mathrm{~kb}$ ) as having multiple methylation profiles (Figure 1F). For comparison, we only identified $6.9 \%$ promoters (1203 of 17450 ) to have DMRs between the two cell-types. Within each promoter window, methylFlow found on average $\sim 55.7$ profiles in the segment with the most potential profiles (Figure $1 \mathrm{G}$ ). We also found that the log of the segment coverage is highly correlated with the log of the number of profiles in a segment $(r=0.75$, Pearson) and the log of the segment length ( $r=0.89$, Pearson) (Supplementary Figure S3a, b). It is highly likely that this increase in patterns is driven by noise since the potential for individual read errors and biological noise increases with coverage as well.

Given that methylation across these regions is associated with expression (29) and that the functional significance of a methylation change at a single $\mathrm{CpG}$ site is usually unclear, it becomes difficult to interpret the number of potential patterns in each segment. Further, since the methylFlow segments are relatively small with a median length of 200-750 bp (36), enumerating the potential combinations of these profiles yields many more potential profiles at each promoter. As an example, there are upwards of $1.7 \times 10^{9}$ possible promoter profiles from combining predicted segments for LARP4B across the $10 \mathrm{~kb}$ region centered at the TSS (Figure 1F). This corresponds to orders of magnitude more profiles than the number of cells used for methylation analysis. As such, though consideration of the spectrum of possible methylation profiles can be of interest, many researchers may want to interpret their data as an average methylation profile for a cell-type in a mixture. Based on the analysis of imprinted regions and this analysis, we thus sought to balance enumerating individual patterns with substantial smoothing to facilitate biological interpretation in our deconvolution strategy.

\section{Number of cell type-specific methylation patterns}

An important consideration in any deconvolution strategy is how many different methylation patterns are expected in a complex mixture. To understand how many different methylation patterns between cell-types would be expected in mixtures of hematologic cell types, we identified all DMRs between nine different hematological cell types in large promoter windows (TSS $\pm 5 \mathrm{~kb}$ ). Next, for a group of cell-types (e.g. GCB, CD4T, monocyte), we considered if the methylation profiles in a given promoter window could reliably distinguish among the cell types, defined as if there were at least one DMR between each pair of cell-types (see examples in Supplementary Figure S4) in the promoter window. To be as inclusive as possible, we did not merge any DMRs that overlapped multiple cell-types. Even using this set of criteria, which likely overestimates the number of genes with distinct DMRs between cell types, we found that in a given promoter window, there are rarely, if ever, four or more distinct DMRs between these cell types (Figure $1 \mathrm{H}$ ). 


\section{DXM design criteria}

Based on these observations, we developed DXM to determine subpopulation methylation profiles spanning a userdefined set of genomic regions (e.g. a $\pm 5 \mathrm{~kb}$ promoter window for expressed genes, CGIs, imprinted DMRs, etc.) and a list of intrasample differentially methylated regions (iDMRs), or regions where allelic subpopulations have differential methylation. Each region is solved separately, reflecting how the number of distinct allelic subpopulation methylation profiles will vary across different regions (e.g. in a mixture of three cell-types, each promoter could have 1-3 different methylation profiles). DXM considers every $\mathrm{CpG}$ in a region for calculation but offers an effective method of smoothing out smaller individual changes without using binning. The resulting methylation profiles solved by DXM are binarized, reflecting how a given allele is either methylated or unmethylated.

\section{DXM performance on simulated idealized mixtures}

We first demonstrated DXM performance in simulated idealized cases of HMEC-CD4+T and HMEC-HCC1954 cell mixtures. To remove potential noise, methylation levels (mCG/CG) were binarized to 0 and 1 for each cell type. For a fixed coverage, unmethylated and methylated counts for each $\mathrm{CpG}$ site were modeled using a binomial distribution. This effectively assumes that errors from inadequate bisulfite conversion and sequencing are negligible relative to the binomial sampling error (see methods for details). As seen in these simulations, DXM performs very well. The number of genes detected to have multiple profiles (i.e. sensitivity) increases with coverage until $\sim 30-90 \times$ depending on the prevalences of the underlying cell-types (Supplementary Figures S5a and S6a). The accuracy of each profile is very high across most coverages (see Evaluation Metrics in methods for details, Supplementary Figures S5b-c and S6b-c), and on average $96.2 \%$ for HMEC-CD4+T cell mixtures and 95.1\% for HMEC-HCC1954 cell mixtures when the coverage is greater than $30 \times$. Together this demonstrates that DXM is generally optimized to only call profiles when it can do so accurately, otherwise it refrains.

\section{DXM identifies allele-specific methylation profiles in $\mathrm{X}$ - linked CGIs and imprinted regions}

To determine whether DXM could identify allele specific methylation profiles in somatic cells, we next considered Xlinked CGIs and imprinted regions. We first applied DXM to deconvolve methylation data from CGIs associated with $\mathrm{X}$-inactivated genes in 14 cell lines and 21 somatic tissues (Figure 2A, B, Supplementary Table S3). On average, we identified differential methylation patterns across $\sim 92 \%$ of the CGIs in females but only $\sim 14 \%$ of the CGIs in males. The variance from the hypothetical result of 0 and 100 is within what is expected due to a normal variation (e.g. age-associated drift) or sex-specific methylation patterns in males (45). For imprinted regions, we first analyzed methylation profiles of imprinted regions at various somatic tissues and cell types (available at REP and BEP, Supplementary Table S4). DXM accurately predicts 2 subpopulations in imprinted regions for $99.0 \%$ (485 out of 490) of imprinted DMRs with average coverage $\geq 10$ in primary cells and at $99.3 \%$ (751 out of 756 ) in primary tissues. We then used DXM to analyze imprinted regions in the human embryonic stem (ES) cell line WIBR3 before and after converting it to a naïve state using $4 \mathrm{i} / \mathrm{L} / \mathrm{A}$ media or doxycycline (DOX)-inducible KLF2 and NANOG transgenes (28). DXM identified multiple methylation patterns at $59 \%$ of imprinted loci for primed WIBR 3 cells, and at less than 10\% of imprinted loci for naïve WIBR3 cells (Figure 2C, Supplementary Table 4). This is in concordance with the expectation that ES cells lose imprints when converted from primed to naïve states (28). Taken together, these results demonstrate the utility of DXM analysis to examine allelic methylation patterns in the context of X-inactivation and genomic imprinting.

\section{DXM accurately solves subpopulation methylation profiles in heterogeneous mixtures}

To determine the effectiveness of DXM in solving deconvolved methylation profiles in cellular mixtures, we generated a series of simulated mixtures by subsampling bisulfite sequencing reads from sorted germinal center B-cells (GCB, from tonsil (24)) and monocytes (from cord blood (24)) at a fixed expected average coverage of $55 \times$. Our simulations included cases where there were more GCBs as well as more monocytes. We initially considered methylation profiles in $\mathrm{a} \pm 5 \mathrm{~kb}$ window around the TSS since this includes the regions where differential methylation most likely impacts gene expression (44). We then applied DXM to deconvolve these mixtures (example output in Figure 3A, Supplementary Figure S7) to assess performance.

We first evaluated the accuracy of DXM-deconvolved subprofiles. Methylation profiles output by DXM were highly accurate for both major and minor subpopulations. DXM correctly identified methylation across 98.5\% of CpGs for promoters with one methylation pattern, 96.2\% for major methylation profiles, and $87.1 \%$ for minor methylation profiles (average performance across all prevalence mixtures for GCB-monocytes, Figure 3B). Similar results were obtained for mixtures generated from sorted CD4+TCD8+T cells from BEP (Supplementary Figure S8) as well as from a mixture of HMEC and HCC1954 cells (Supplementary Figure S9). Increasing sequencing coverage up to 88x did not substantially impact reconstruction accuracy (Supplementary Figure S10a) or profile assignment (Supplementary Figure S10b), consistent with earlier simulations (Supplementary Figures S5 and S6).

We next examined whether DXM deconvolved profiles could be assigned to the appropriate cell type at promoter regions with distinct cell-type specific methylation profiles (i.e. promoter regions that contain a DMR identified between the two cell types). For each promoter, DXM-solved subpopulation methylation profiles were assigned to the closest reference cell type. As expected, the percent of profiles assigned to the GCB cell type in the major subpopulation increases as the ratio of GCB reads increases (Figure $3 \mathrm{C}$ ). The inverse relationship can be seen for the minor subpopulation. DXM performs best when the minor subpopulation comprises $20 \%$ or more of the mixture. This might be expected for our simulations, since in a $55 \times$ cov- 
A

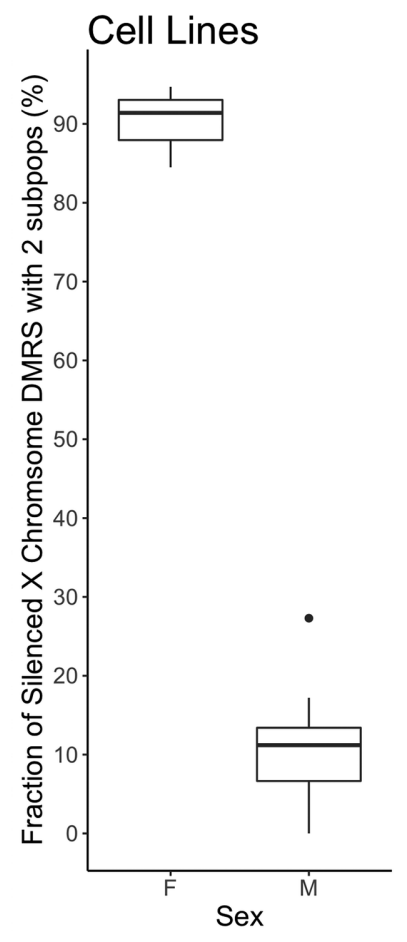

B

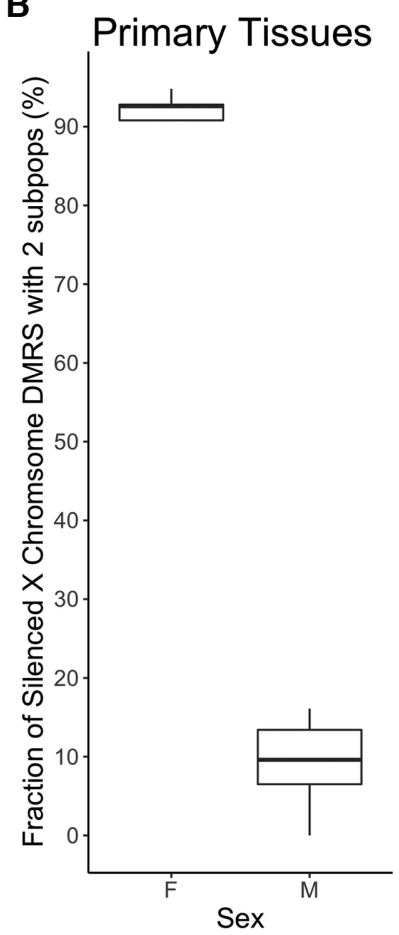

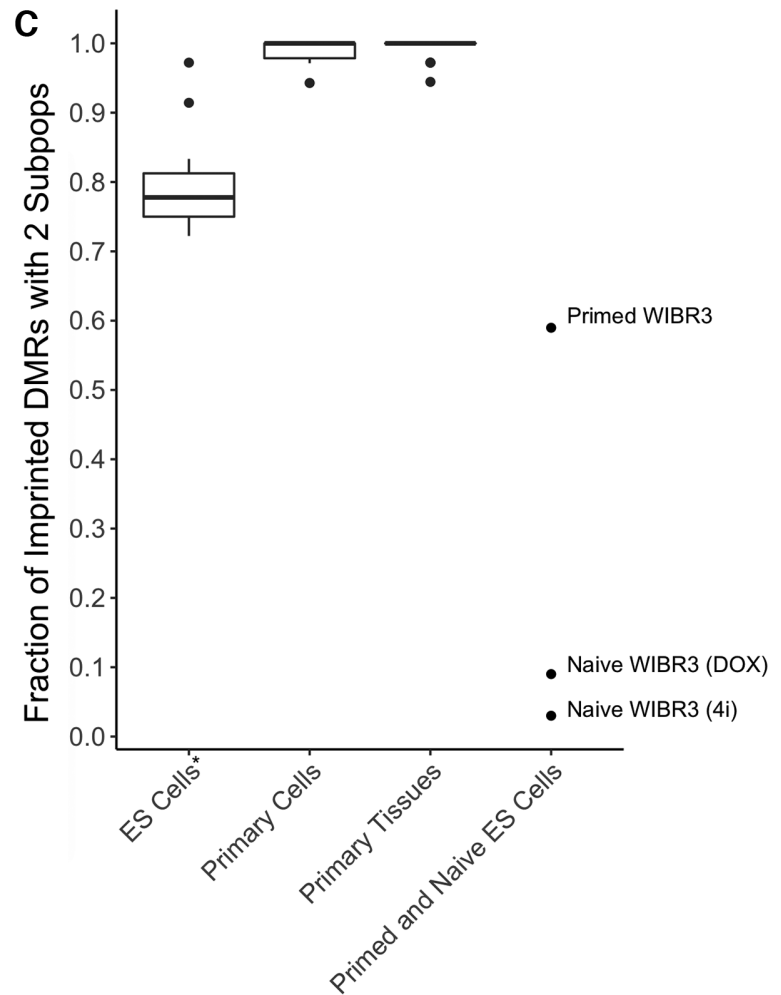

Figure 2. DXM accurately deconvolves methylation profiles at X-linked CGIs and imprinted regions. (A, B) DXM was used to deconvolve CGIs identified on the X chromosome in (A) cell lines and (B) primary tissues. DXM finds multiple methylation patterns in a median of $91.4 \%$ of female cell lines and $92.6 \%$ of female primary tissues but only in $11.2 \%$ male cell lines and $9.6 \%$ in male primary tissues. (C) DXM accurately deconvolves methylation profiles in imprinted regions. DXM was used to deconvolve CGIs identified on the X chromosome in cell lines and primary tissues from the Roadmap Epigenomics and Blueprint projects. ES Cells* include ES cells, ES derived cells, and iPSCs, which are known to lose imprints. DXM accurately predicts two subpopulations in imprinted regions for $99.0 \%$ (485 out of 490 ) of imprinted DMRs with average coverage $\geq 10$ in primary cells and at $99.3 \%(751$ out of 756 ) in primary tissues. DXM finds fewer patterns of imprinting $(\sim 76 \%)$ in ES cells as expected. Full lists of the samples used are in Supplementary Tables S3 and S4. Data from primed WIBR3 ES cells as well as naïve WIBR3 cells converted using 4i/L/A or DOX (doxycycline-inducible KLF2 and NANOG transgenes) is from $(28)$.

erage mixture, a $10 \%$ subpopulation corresponds on average to only 5.5 reads. For some promoters, especially when the prevalence of the minor subpopulation is less than $20 \%$, the minor methylation profiles output by DXM are closer to the true major subpopulation reference profile, rather than the minor population reference (Supplementary Figure S11a, b). This observation likely reflects how intrinsic variation in typical methylation data from single sorted cell types at fully methylated and unmethylated $\mathrm{CpG}$ sites, the purity of the sorted cell populations, and the number of CpGs with differential methylation between cell subpopulations masks the ability of deconvolution approaches to detect low prevalence subpopulations (Figure 1C-E). Even with the beta-binomial error models used by DXM, noise in individual datasets can substantially affect the smallest allele fraction that can be detected.

\section{DXM predictions for more than two subpopulations}

We further sought to evaluate DXM performance for mixtures with more than two subpopulations. We applied DXM to a $55 \times$ simulated mixture of $\mathrm{CD}^{+} \mathrm{T}$ : GCB:monocytes (10:25:65). We focused on only 3 subpopulations since we found very few gene promoters with more than that many distinct profiles (Figure 1H, Supplementary Figure S12a). The 496 genes that have a DMR between each pair of cell types in this mixture showed enrichment in several immune pathways, which is expected from ontology analysis. DXM identified multiple methylation profiles at 434 of the 490 $(88.5 \%)$ promoters expected to have three methylation profiles, but $342(79 \%)$ of these promoters were solved with only two profiles instead of three (Supplementary Figure $\mathrm{S} 12 \mathrm{~b}$ ). This suggests that in those 342 promoters, DXM identified subpopulation methylation differences but could not resolve them into three distinct profiles. For the deconvolved promoters, DXM had high accuracy for both the major $(95.9 \%)$ and minor $(87.4 \%)$ profiles (Supplementary Figure S12c). This accuracy resembles what was seen for $k=2$ simulations (Figure 3B). For the 57 promoters solved with three methylation profiles, DXM had high accuracy for the first $(96.0 \%)$, second $(87.0 \%)$ and third $(77.6 \%)$ profile (Supplementary Figure S12d). Given that on average only 5.5 reads are supporting the lowest subpopulation, it is thus not surprising that DXM frequently does not detect this subpopulation, and when it does it has lower accuracy. Taken together, DXM can accurately deconvolves subpopulation methylation profiles in mixtures with three subpopulations. 

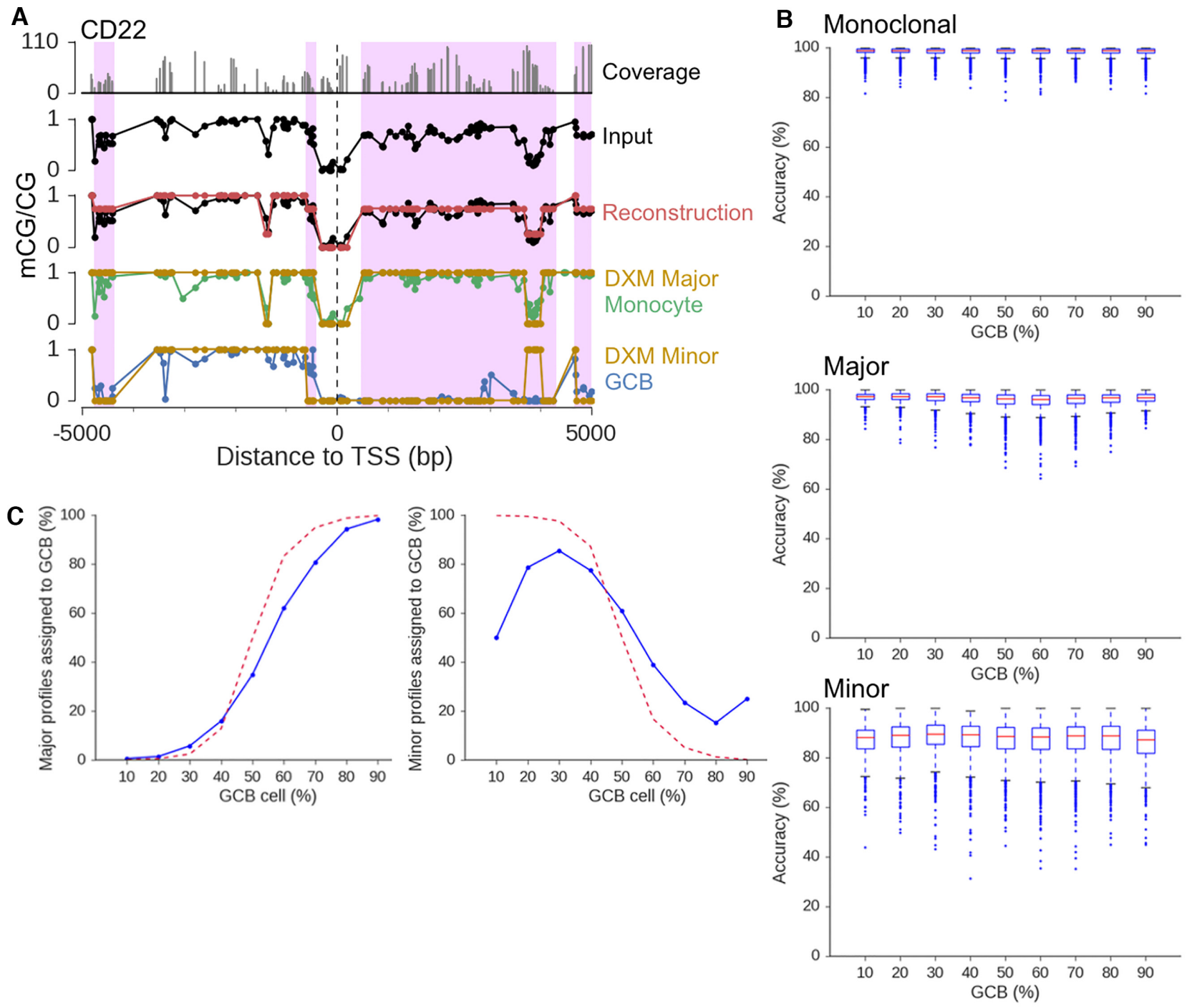

Figure 3. DXM accurately solves for subpopulation methylation profiles in heterogeneous mixtures. (A) DXM solution for CD22 for a $55 \times$ coverage simulated mixture of monocytes and GCB (35:65). (B) Accuracy of DXM methylation profiles with respect to reference GCB or monocyte profiles for promoters where DXM identified the same number of methylation profiles as expected by DSS. (C) Number of gene promoter profiles assigned to GCB cells (blue) across 55x coverage mixture simulations with different GCB prevalences. The dotted red line indicates the number of $\mathrm{CpGs}$ with more subsampled reads from GCB cells than monocytes.

\section{DXM accurately deconvolves subpopulation methylation pro- files at enhancers}

We applied DXM to a list of enhancer regions defined based on H3K27Ac and H3K4me1 ChIP-Seq data that are expected to be active in either GCB cells or monocytes $(46,47)$. We performed similar read-level simulation mixtures to those above and found that DXM performed well with median reconstruction accuracies across all prevalences of $>99 \%$ if the same methylation pattern is present, $96.1 \%$ for the major subpopulation, and $80.0 \%$ for the minor subpopulation (Supplementary Figure S13). This performance is maintained across all mixture conditions. This suggests that the mathematical framework of DXM is sound, and that it can be applied to other genomic regions such as enhancers.
DXM outperforms existing bisulfite sequencing deconvolution algorithms

We compared DXM with methylPurify (22), an expectation maximization-based approach developed to separate normal and tumor cell profiles from a heterogenous mixture. MethylPurify first identifies a series of most informative $300 \mathrm{bp}$ bins that show intermediate methylation. Using those bins, it then predicts the prevalence of the two underlying cell types and then estimates the average methylation in each cell type across that bin. Data from adjacent bins can then be stitched together to make a profile. MethylPurify has several limitations relative to DXM: it is only compatible with human genome versions hg18 and hg19, it appears to have been only benchmarked at CGIs, it assumes there are only two underlying cell types, and 
does not natively work with reads that are $100 \mathrm{bp}$ or longer. MethylPurify also cannot distinguish methylation changes at individual $\mathrm{CpG}$ sites since it uses a binning scheme (default of $300 \mathrm{bp}$ ) to average all methylation changes within a bin (example in Figure 4A). We first generated a $20 \times$ coverage simulated mixture of HMEC:HCC1954 cells (35:65) and applied DXM and methylPurify to deconvolve the sample. We chose this data since it was used to originally benchmark methylPurify, and because the GCB:monocyte mixtures used above had longer read lengths and would not natively run with methylPurify. For this mixture, both methylPurify and DXM accurately solved methylation profiles of the underlying cell types (methylPurify $98.9 \%$, DXM 97.8\%). DXM identified i-DMRs at 58\% (15009/26 078) of the most informative bins (mib) predicted by methylPurify, while regions specific to either method tended to cover fewer CpGs $(P<0.001$, Cohen's $d>0.8$, Supplementary Figure S14a, b).

Next, we edited methylPurify's code to allow reads longer than $100 \mathrm{bp}$ and compared DXM with methylPurify across mixtures of GCBs and monocytes where coverage was fixed at 40x (Figure 4B, C). While the accuracy of DXM and methylPurify is similar for the major subpopulation, DXM's accuracy for the minor subpopulation is much higher. We found that DXM performs well across CGI and promoter windows, with average accuracy of $\sim 98 \%$ in major subpopulations and $\sim 84 \%$ across minor subpopulations. methylPurify does well with major subpopulations (average of 99\% accuracy), but performance across minor subpopulations falls below $50 \%$ in most mixtures. MethylPurify had very low accuracy in regions identified by both it and DXM, suggesting the difference is not strictly due to methylPurify trying to deconvolve a more difficult set of regions. We suspect methylPurify may underperform because it misestimates the fractions of the cell populations for these mixtures; it estimated that the prevalence was 0.055 for the minor cell type for most mixtures (Supplementary Figure S14c). Taken together, we conclude that while DXM performs similarly to methylPurify at accurately deconvolving DNA methylation profiles for major subpopulations, DXM is more robust at accurately deconvolving the minor profile across a variety of types of cell mixtures and regions.

Given an appropriate region signature, DXM accurately solves prevalence of underlying cell-types in heterogeneous mixtures

While the primary goal of DXM was to deconvolve subpopulation level methylation changes, we next sought to determine whether DXM could also accurately estimate the prevalence of allelic subpopulations. The analogous problem for calling genetic subclones is based on the general assumption that genomic sequencing analysis of a sample containing a subpopulation with $20 \%$ prevalence should yield a peak in the distribution of variant allele frequencies (VAFs) around $20 \%$, which reflects the mutations associated with the subclone. Similarly, if there are $\mathrm{CpGs}$ that only are methylated in a certain subclone that comprises $20 \%$ of the sample, the expected fractional methylation of these CpGs in the sample would be $20 \%$. However, in a 20:80 mixture of GCBs and monocytes, we found that the methylation distribution of all $\mathrm{CpG}$ does not even have a minor peak near 20\% (Supplementary Figure S15a). Thus it would be difficult in practice to consider all $\mathrm{CpGs}$ to determine absolute prevalence. Instead the better approach is to select a subset of $\mathrm{CpGs}$ that differ between the clones and choose those. We first considered only $\mathrm{CpGs}$ that were found in i-DMRs (Supplementary Figure S15b), and found that the distributions still did not show clearly defined peaks around $20 \%$ and $80 \%$, and in practice prevalence estimates were poor (data not shown). We next defined a signature based on gene promoters containing DMRs that are differentially expressed between GCB cells and monocytes in a different set of samples (see methods). Using this region signature, DXM predictions have very high agreement with expected prevalence when the expected prevalence of the minor subpopulation is between 15-40\% (Supplementary Figure $\mathrm{S} 15 \mathrm{c}, \mathrm{d}$ ). This suggests that given an outside signature of regions that show differential methylation between samples, DXM can accurately identify subpopulation prevalences.

\section{Comparison of i-DMRs from simulated mixtures and DMRs between cell types}

We next examined the degree of concordance between DXM i-DMRs predicted from simulated cell mixtures and DMRs identified from the underlying cell types using the same GCB-monocyte mixtures as above. Our goal was to determine if DXM was reasonably calling i-DMRs. DXM on average found an i-DMR between underlying subpopulations in about $64 \%$ of all DMRs (Supplementary Figure S16). This is consistent with what would be expected given the wide-variability seen in different DMR callers (48-50). Across all simulated mixture ratios, we found that DXM predicted a much larger number of i-DMRs for GCBmonocyte mixtures relative to the number of DMRs DSS identified between each underlying cell type (e.g. for a 30:70 mixture, 19755 i-DMRs and 3012 DMRs, or 5722 versus 2187 gene promoters).

The DMRs that were not identified by DXM tended to be shorter and have fewer CpGs (Supplementary Figure S17). Shorter DMRs with fewer CpGs have less empirical support and whether they are called DMRs tends to be more variable across DMR callers. Additionally, we found that increasing sequencing coverage did not improve this overlap, though it increased the total number of i-DMRs and DMRs found (Supplementary Figure S18a). However, given the coordinates of where to expect these DMRs, we next isolated each 'missed' DMR and reran DXM for just the DMR instead of across the full $\pm 5 \mathrm{~kb}$ promoter window. With this updated region definition, we found that on average, DXM identified as i-DMRs $\sim 86 \%$ of these previously 'missed DMRs' (Supplementary Figure S18b). Taken together, this highlights how DXM and DSS use different approaches to smoothing the data and locating DMRs but can recapitulate similar results.

DXM identifies substantial subpopulation methylation profiles in sorted cell types

We next sought to understand why DXM predicted many i-DMRs in regions that did not have a DMR between the 


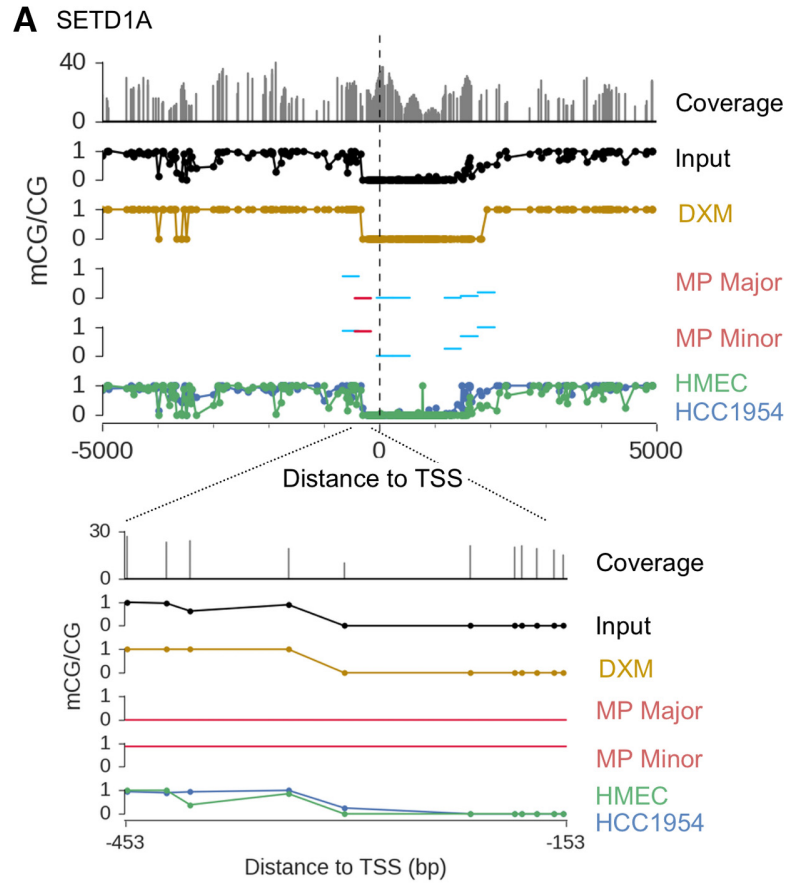

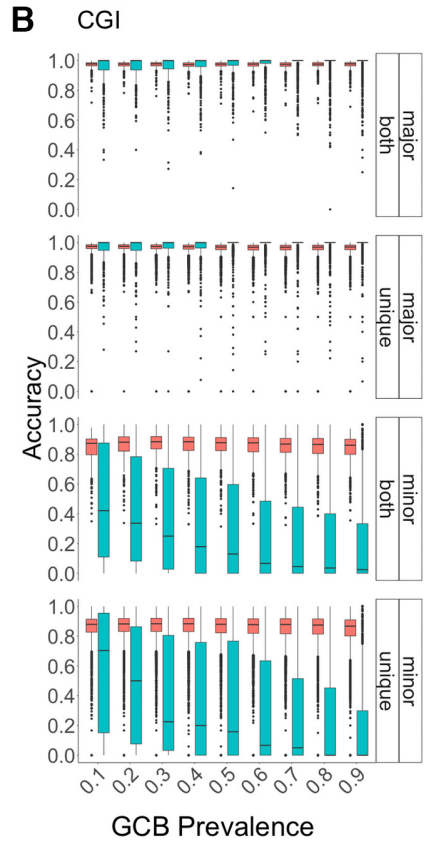

白 DXM
C TSS $+/-5 \mathrm{~kb}$

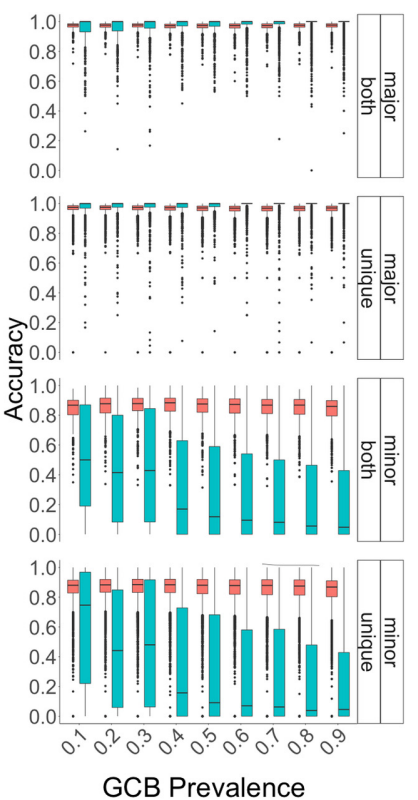

追 MethylPurify

Figure 4. DXM outperforms existing methods. (A) methylPurify (MP) and DXM outputs for SETD1A in a 20× coverage HMEC-HCC1954 mixture (35:65). Both all (sky blue) and most informative (crimson) bins as determined by MP are depicted. Despite agreement between HMEC and HCC1954 profiles, MP separates this region into two profiles (likely due to the fixed bin size). DXM, however, predicts only one profile. (B, C) Reconstruction accuracy is plotted for $(\mathbf{B})$ CGIs and $(\mathbf{C})$ promoter windows (TSS $\pm 5 \mathrm{~kb}$ ). Regions where both methods identified multiple methylation profiles are labeled 'both,' and regions where only one method identified multiple methylation patterns (DXM i-DMR, methylPurify informative bin) are plotted as 'unique.' Whether or not a region was identified by 'both' methods or by one method alone ('unique') did not affect accuracy. While DXM accurately deconvolves methylation profiles across CGI and promoter windows for both major and minor subpopulations, methylPurify only accurately deconvolves profiles for the major profile.

cell-types. Plotting the distribution of methylation levels (mCG/CG) for the reference GCB and monocytes shows that these levels are often similar between cell-types but in an intermediate methylation state (e.g. 30\% methylation in GCB and 30\% in monocytes) (Figure 5A). One interpretation is that for these loci, there are distinct DNA methylation patterns in subpopulations, but these subpopulations differ from the expected reference cell types as might be defined from sorting. Supporting this is the observation that when we applied DXM to only GCB cells or only monocytes alone, we found that $70 \%$ of the DXMspecific i-DMRs predicted in the GCB:monocyte mixture were also detected as i-DMRs within only GCB cells or only monocytes. However, it is unlikely that the multiple patterns found within these cell types represent what is traditionally referred to as biological heterogeneity (e.g. multiple cell subtypes within a sorted cell type such as light and dark zone GCBs or classical/non-classical monocytes), since the sub-profiles are mostly shared by two distinct sorted cell types: GCBs and monocytes. For instance, when we look at i-DMRs that lie within nine total sorted cell types (CD4+ $\mathrm{T}$, CD8+ T, eosinophil, erythroblast, GCB, hematopoietic multipotent multiprogenitor, megakaryocyte, monocyte, osteoclasts), we detected 840 promoters with an iDMR within each of the nine cell-types and $\sim 500$ genes where five cell-types exhibited the i-DMR (Figure 5B). The distribution of methylation levels of $\mathrm{CpGs}$ in these common
i-DMRs is not centered around $50 \%$, suggesting that they are likely not due to imprinting or allele specific methylation (Figure 5C).

We next sought to verify that the extensive heterogeneity we observed was likely due to multiple cells in these sorted populations with distinct biological states. We thus ran DXM on individual sorted cell types amongst nine hematologic cell-types (CD4T, CD8T, eosinophil, erythroblast, GCB, hematopoietic multiprogenitor cells, megakaryocytes, monocytes, osteoclasts). Ontology analysis indicates that i-DMRs that are shared across all nine cell-types are enriched for genes associated with cadherin-domain proteins (Supplementary Table S5). Cadherins are critical to celladhesion (51), which regulates many aspects of leukocyte function, including extravasation and vascular permeability for circulating leukocytes (52). This suggests there is a methylation signature across these genes that could be associated with reduced cadherin expression, which could reflect decreased tendency of a particular cell to adhere or a change in active state for the cell (e.g. circulating vs extravasating). Further, because cell-adhesion is a pathway common to all cells, this is consistent with the finding that there are multiple methylation profiles within sorted cell types that may not correspond to traditional cell subtypes but certainly could represent distinct biological states. Thus, DXM-specific iDMRs likely represent a separate class of relevant subpopulation methylation events. 
A

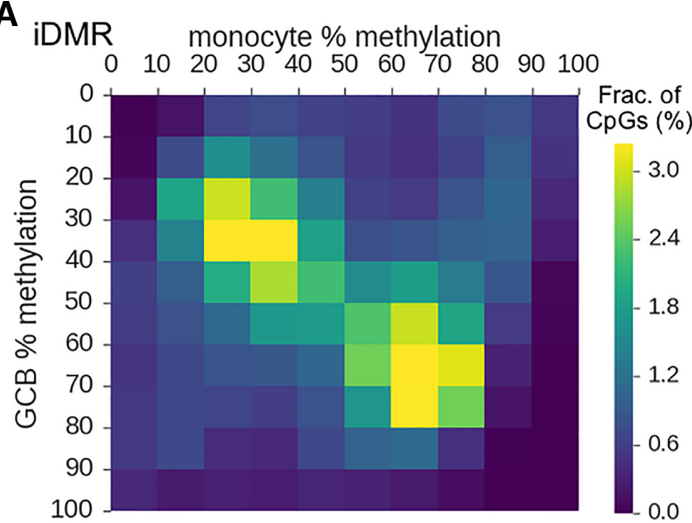

B

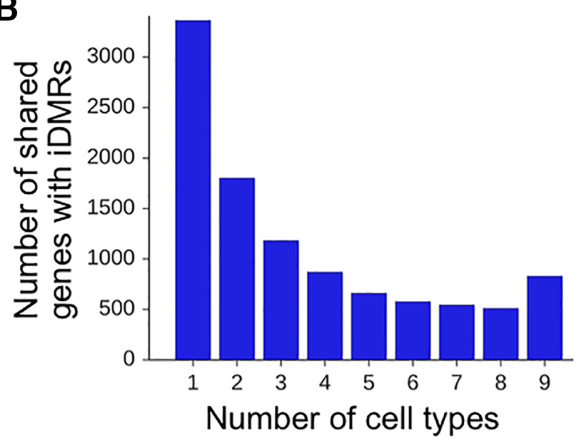

DMR monocyte \% methylation

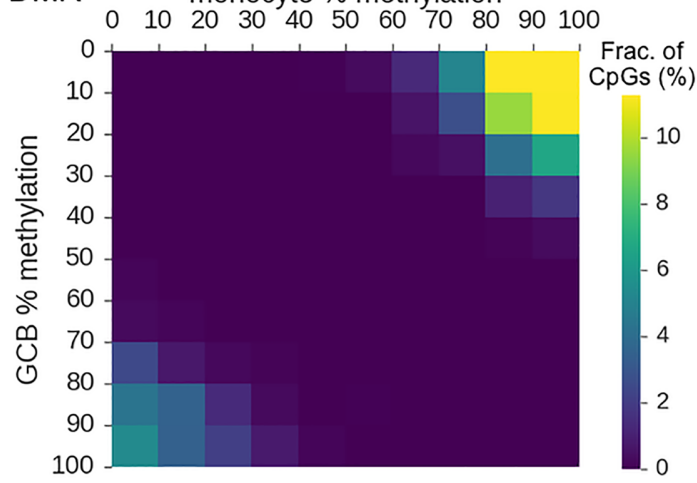

$c$

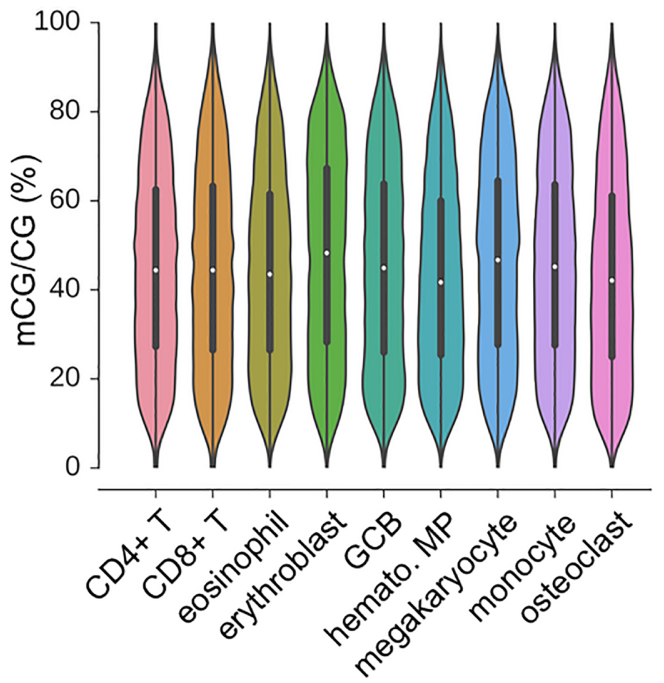

Figure 5. DXM identifies many i-DMRs within sorted cell types. (A) i-DMRs and DMRs identified in a 55× mixture of GCB:monocytes (30:70) have different methylation profiles of the underlying reference GCB cells and monocytes. For instance, many i-DMRs are found with 30-40\% methylation in both GCB and monocytes. (B) The number of gene promoters with i-DMRs that are shared between different numbers of sorted cell types is shown. There are substantial numbers of i-DMRs that are both unique to individual cell-types, as well as shared across many cell types. The cell types used are listed in (C). (C) Fractional methylation distribution of $\mathrm{CpGs}$ in i-DMRs for sorted cell types.

\section{DXM predicts subpopulation differences in methylation in primary DLBCL samples}

We next experimentally validated DXM predictions from Agilent Methyl-Seq analysis of lymph node biopsies from four DLBCL patients (sample characteristics are in Supplementary Table S1). Single cell suspensions from biopsies were split in half. Half of the cells were sorted by fluorescence activated cell sorting (FACS) into CD4+ T and CD19+ B cell populations, the expected major cell types present in DLBCL lymph node biopsies. The other half was subjected to Agilent Methyl-Seq, which is a bisulfite sequencing approach that enriches for gene promoter regions using capture probes similar to exome capture techniques (53). We obtained an average of $>54 \mathrm{x}$ coverage across $\sim 4.8$ million $\mathrm{CpGs}$ for all samples, including $>58 \times$ coverage for $>2$ million out of $\sim 3.5$ million $\mathrm{CpGs}$ in the $10 \mathrm{~kb}$ region around the TSS (Supplementary Table S6). CGIs and the $\pm 5 \mathrm{~kb}$ region at the TSS were hypermethylated in DLBCLs relative to normal cell types, while proximal regions were hypomethylated as has been observed in prior DLBCL studies (5) and are typical in most cancers (54) (Figure 6A, B).
After running DXM on each DLBCL sample, we first compared DXM-solved estimates (using a gene signature from normal $\mathrm{CD}^{+} \mathrm{T}$ and $\mathrm{GCB}$ cells as above) of the relative proportion of $\mathrm{T}$ and $\mathrm{B}$ cells, to $\mathrm{T}$ and $\mathrm{B}$ cell proportions determined by FACS and to clinical flow cytometry. Of note, clinical flow cytometry for $\mathrm{T}$ and $\mathrm{B}$ cells was performed on an adjacent section of the same lymph node at the time of biopsy, while FACS was performed from the same single cell suspension used for Agilent Methyl-Seq. We found good agreement between DXM-estimated prevalence and FACS results (on average, fractional prevalence estimates were within 0.0875 of each other, B-cells shown in Figure 6C). Clinical flow data was consistent with FACS and DXM for all but one DLBCL sample (DLBCL 2). DXM results likely are more consistent with FACS sorting because clinical was obtained from a different section of the biopsy, and DLBCL lymph nodes are known to be heterogeneous.

Next, we estimated the accuracy of DXM subpopulation methylation profiles for the gene signature between normal $\mathrm{CD}^{+}{ }^{+} \mathrm{T}$-cells and normal GCB-cells. For these 2,454 genes, DXM identified differential methylation in $901-1141$ pro- 
A
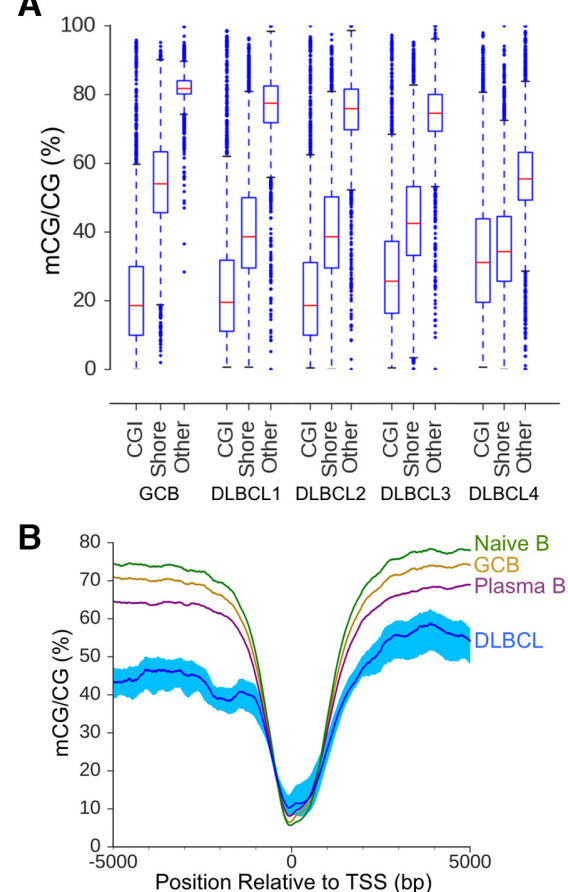

C

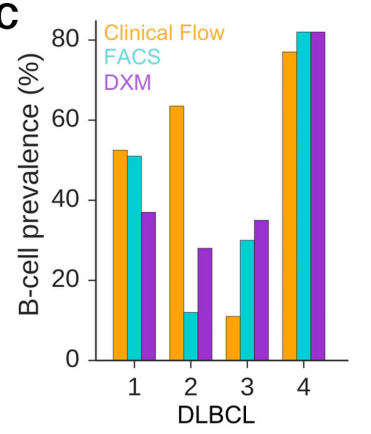

D

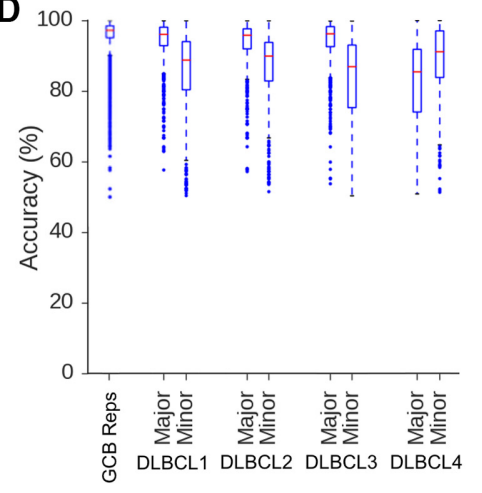

E

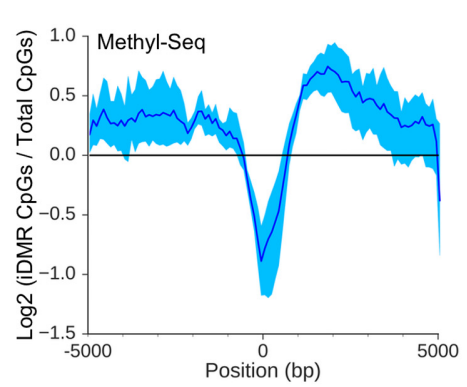

$\mathbf{F}$

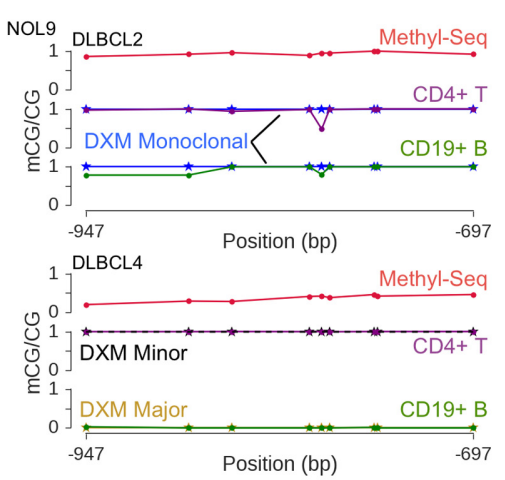

SPIB

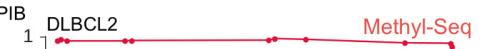

0 . 0

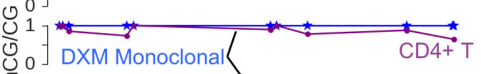

U 0 - DXM Monoclonal

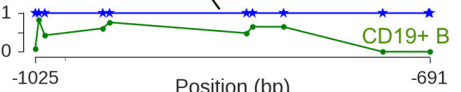

-1025
DLBCL

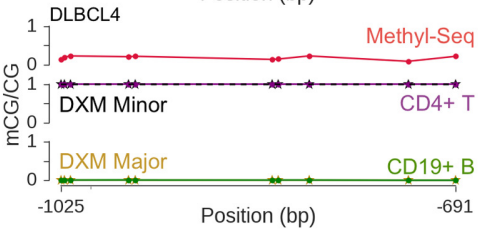

G

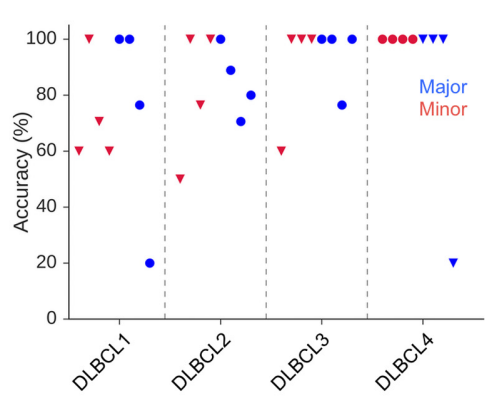

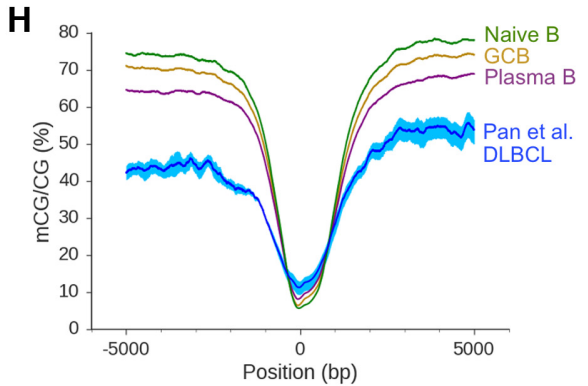

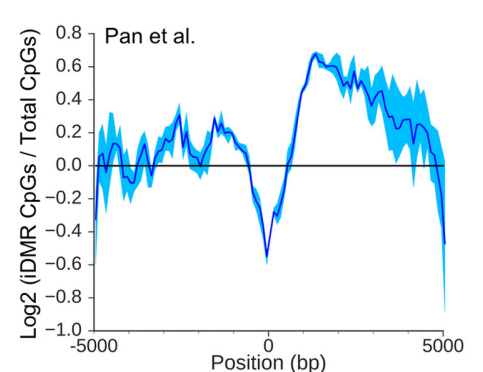

J

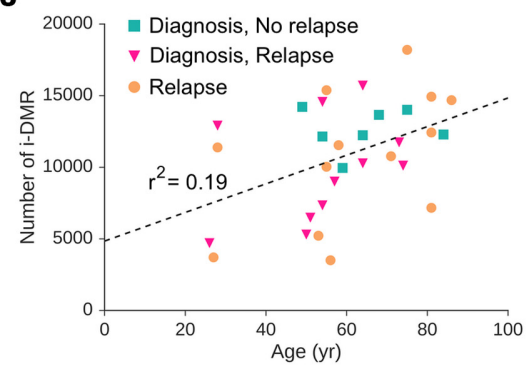

K

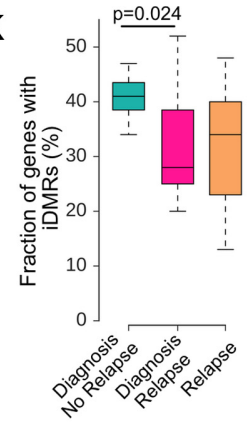

Figure 6. Experimental validation of DXM predictions in DLBCLs. (A) Distribution of methylation for CpGs in CGIs, CGI shores or other regions for four DLBCL lymph node biopsies profiled by Agilent Methyl-Seq. (B) Meta-gene analysis (with respect to the TSS) of the four DLBCL samples and reference cell types show hypermethylation at and hypomethylation up- and down-stream of the TSS. Dark blue denotes the average sample profile, with light blue indicating the maximum and minimum range. (C) Percentage of B cells as measured by clinical flow (orange), FACS (light blue), and DXM (purple). (D) Accuracy of DXM predictions for each sample at 2454 gene promoters with DMRs identified between normal CD4 ${ }^{+}$T-cells and GCB cells. (E) i-DMRs are enriched $500 \mathrm{bp}-3 \mathrm{~kb}$ downstream of the TSS. Shading as in B. (F) Targeted bisulfite sequencing results for NOL9 and SPIB for two DLBCL samples. Results for additional genes and samples are in Supplementary Figure S19. Methyl-Seq input data (red), DXM predictions (monoclonal blue, major profile gold, minor profile black dash), sorted CD4+ T-cells (purple), sorted CD19+ B-cells (green). (G) Accuracy of DXM predicted profiles in a targeted bisulfite sequencing experiment for four DLBCL samples across four loci (NOL9, SPIB, CD22, BCL2L1) for sorted CD19+ B-cells and CD4+ T-cells. Blue $=$ major subpopulation, Red $=$ minor subpopulation, triangle $=\mathrm{B}$-cell, circle $=\mathrm{T}$-cell. $(\mathbf{H})$ Meta-gene analysis (with respect to the TSS) of the 31 DLBCLs from Pan et al. (5) and reference cell types show hypermethylation at and hypomethylation away from the TSS. Shading as in B. (I) i-DMRs are enriched $500 \mathrm{bp}-3 \mathrm{~kb}$ downstream of the TSS in 31 samples from Pan et al. Shading as in B. (J) Number of i-DMR detected in the 31 DLBCL samples with respect to patient age $\left(r^{2}=0.19\right)$. (K) Fraction of gene promoters with an i-DMR for DLBCL samples at diagnosis (blue-green, $\left.n=11\right)$, at diagnosis that did not have future relapse (pink, $n=7$ ), and at relapse (orange, $n=13$ ). 
moters with an average accuracy of $91 \%$ for major subpopulations and $86 \%$ for minor subpopulations, which approaches the levels observed during benchmarking (Figures 3B and 6D). The clear outlier is DLBCL 4, which has substantially more B cells than T cells (Figure 6C) and shows greater CGI hypermethylation. Thus, the major population of DLBCL 4 is likely comprised of malignant B-cells, whose methylation profiles differ substantially from the normal GCBs used as a reference to estimate the accuracy, causing an artificially low estimated accuracy. For each sample, DXM found 3785-6790 gene promoters with at least one iDMR (Supplementary Table S6). While i-DMRs were not enriched in CGIs or CGI shores (mean O/E $=1.01$ for CGIs and 0.94 for CGI shores, $P=0.976$ ), we did observe more i-DMRs in the region located 500-3000 bp downstream of the TSS (Figure 6E), which has been found to strongly associate with expression changes (29).

To experimentally validate DXM profile predictions, we conducted targeted bisulfite sequencing in sorted $\mathrm{CD} 4^{+} \mathrm{T}$ cells and $\mathrm{CD} 19^{+} \mathrm{B}$-cells from each sample. We selected four loci that regulate B-cell specific function (SPIB (55), CD22 (56)) or are commonly mutated in DLBCL (BCL2L1 (57), NOL9 (58)) and for which DXM predicted an i-DMR in at least one but not all samples. DXM predicted the correct number of underlying methylation profiles (one or two) across each locus and each patient (example in Figure 6F, data for all 16 cases found in Supplementary Figure S19). Additionally, DXM predictions for subpopulation methylation profiles are recapitulated in sorted cell types with an average accuracy of $84.7 \%$ (Figure 6G).

As proof-of-concept, we applied DXM to a publicly available cohort (Cohort 1 in (5)) of 31 DLBCLs (11 paired diagnosis-relapse, 1 of which had 3 relapses, 7 with no relapse) profiled by enhanced reduced representation bisulfite sequencing (eRRBS). These samples exhibit a similar gain of methylation at the TSS and loss of methylation outside the $2 \mathrm{~kb}$ surrounding the TSS (Figure 6H). DXM identified 3708-18 200 i-DMRs (average of 10 826) for these samples (Supplementary Table S7). i-DMRs were frequently found 500-3000 bp downstream of the TSS (Figure 6I) as seen with our DLBCLs (Figure 6E). We observed a weak correlation between age and the number of i-DMRs detected for each sample (Figure $6 \mathrm{~J}, r^{2}=0.19$ ). This is suggestive that some i-DMRs may be caused by age-associated drift in DNA methylation patterns (43). Additionally, we found that patients presenting with fewer gene promoters with iDMRs at diagnosis had higher rates of relapse (Figure 6K).

\section{DISCUSSION}

We present DXM, a computational method to deconvolve methylation sequencing data from a heterogeneous sample into its major allelic subpopulations and their associated methylation profiles. Importantly, DXM does not require explicit prior knowledge of the expected cell types or number of subpopulations to consider. When using DXM, one consideration is that DXM solves for allelic profiles, which we then interpret as corresponding to unique subpopulations. As such, care must be taken in interpreting DXM results in regions with allele-specific methylation or copy number variants. The same is true for any deconvolution strategy. An additional consideration is that DXM does not consider 5-hydroxymethylation $(5 \mathrm{hmC})$, which may impact interpreting results in samples with high levels of $5 \mathrm{hmC}$, such as neuronal cell types (59).

If given an appropriate set of regions that are known to show variation in the cell types, DXM can accurately measure the allelic fraction of each cell-type. One potential advantage of using DXM to detect cell prevalences is that DNA methylation is highly stable even in cryopreserved samples, especially relative to RNA and protein. Further, malignant cells are often more susceptible to cell death caused by delays related to specimen transport and cryopreservation (Payton, unpublished observations), which could potentially affect clinical flow and FACS. However, additional future testing is required to determine whether a methylation-based strategy using DXM detects cell prevalences more accurately than these standard approaches. As a pseudo-reference-free method, DXM does not require reference profiles for each cell-type. Instead, DXM uses generalized model parameters to identify subpopulation level methylation profiles. However, if reference profiles are available, they can still be useful to interpret DXM results.

To ensure we did not overfit DXM model parameters, we used independent datasets for all training and evaluation steps. While we have demonstrated that DXM can accurately deconvolve DNA methylation profiles for a variety of genomic features in the case studies above, it is possible that retuning DXM parameters (e.g. transition probabilities) for genomic compartments or for individual datasets could improve accuracy. This is likely true for samples with extreme methylation changes such as caused by mutation in a DNA methyltransferase (e.g. DNMT3A in an AML patient (60) or treatment with demethylating agents (61)).

A major challenge of genomic methylation data analysis is determining what constitutes a biologically meaningful methylation change, or a distinct methylation profile. DXM takes an approach to regularize small differences in methylation between two profiles to report a small number of smoothed profiles. This interpretation is used frequently for imprinting control regions, where partially methylated alleles are considered as biological noise, or drift from a true pattern rather than a functionally different state. A similar assumption underlies DMR-callers. The differences between DSS-called DMRs and i-DMRs in artificial mixtures are similar to those observed between different DMRcallers (62). DMR-callers rely on several important userdefined parameters such as the minimum length, minimum number of $\mathrm{CpGs}$, or minimum difference in methylation between samples, that can severely affect the number and type of DMRs in an analysis (30). Despite this, DMR callers are incredibly useful and are part of every common workflow for DNA methylation analysis. The same should be true for DXM i-DMRs.

DXM deconvolves methylation profiles over user-defined regions. Here we have demonstrated how these regions could be CGIs, promoters, enhancers, or imprinted DMRs, but in theory DXM could be applied to any set of genomic regions. When defining regions however it is important to consider how region size will affect the results. As regions get larger and include more $\mathrm{CpGs}$, the number of $\mathrm{CpGs}$ that must have a change in methylation in order to be solved as 
a distinct methylation profile by DXM will also increase. This can be seen by comparisons between DXM and DSS, where many of the DMRs identified by DSS were recapitulated by DXM when given a more specific domain to consider. This can be offset by increased sequencing depth, as the 'resolution' (number of methylation profiles solvable by DXM) of that region increases with increased coverage. While it is possible that with sufficient depth one could try to solve a global methylation profile across an entire chromosome, this would be difficult in practice given gaps in most genome assemblies and frequent large repetitive DNA sequences (e.g. simple repeats or transposons) that complicate obtaining high quality methylation data.

One surprising result from this analysis is that even sorted cell types have a substantial number of intrinsically intermediate methylated regions, or genomic intervals that are not fully methylated, unmethylated or imprinted. Intermediate methylation states can be both cell-type specific and conserved (63). Our results show that even in sorted cell types there is extensive intermediate methylation. One contributing factor is that cells are sorted on a small number of markers and likely contain multiple subsets within them. However, the substantial number of partially methylated reads in imprinted DMRs suggest that this is not the only contributing factor. This extensive intermediate methylation complicates deconvolution efforts, particularly reference-free cell prevalence determination. This suggests that there are fewer CpGs whose methylation is 'cell-type specific' as compared to $\mathrm{CpGs}$ with intermediate methylation levels in an individual cell type.

The origin of this intermediate methylation is not completely understood but likely includes several previously described phenomena including lowly methylated regions (LMRs) and partially methylated domains (PMDs). Initial reports have demonstrated that LMRs, relatively short non-CpG island DNA segments with low levels of methylation, frequently arise from the binding of transcription factors $(64,65)$. However, we find near equal levels of highly and lowly methylated DNA methylation in i-DMRs indicating that while LMRs could contribute to the i-DMRS we observe in individual cell types, they cannot fully explain them. PMDs are broad regions of lower methylation, are commonly found in cancers and to a limited degree in somatic cell types, and are associated with hypervariability (66). Cell-type specific PMDs are predicted by CpG density in addition to late replicating/lamina associated domains (67). Given the lack of association of i-DMRs with CpGdensity, PMDs could contribute to the i-DMRs but are also unlikely to explain them all.

Lastly, we have validated that subpopulation methylation profiles predicted by DXM are recapitulated in relevant sorted cell subpopulations in DLBCL samples. As proofof-concept, we have used DXM to identify subpopulation methylation profiles in DLBCL that may correlate with patient prognosis. Since gene promoters with i-DMRs represent multiple methylation profiles in a sample, it is possible that the number of gene promoters with i-DMRs in a sample serves as a proxy of the subclonal heterogeneity. Based on our reasoning, samples at diagnosis with fewer iDMRs might represent a more clonal disease, which could be more aggressive and more likely to relapse. Since relapse samples go through many changes, including a bottleneck where many of the subclones in the diagnosis sample are lost due to treatment and subsequent expansion of new subclones, as expected there was no clear relationship between the number of $i-D M R s$ at diagnosis and at relapse. Interestingly, a previous analysis of methylation heterogeneity in this cohort found that samples at diagnosis with more epipolymorphisms, regions where epialleles of four or more adjacent $\mathrm{CpG}$ sites exhibit high entropy, are more likely to relapse (5). However, since epialleles describe a different intrasample methylation phenomenon than i-DMRs, it is not surprising that samples at diagnosis with fewer i-DMRs are more likely to relapse. While our initial findings based on 18 patients need to be validated in a larger cohort, they illustrate how DXM could be applied to analyze subpopulation methylation profiles in cancer samples.

In summary, here we have developed a new method DXM to accurately deconvolve methylation profiles of allelic subpopulations in genomic-bisulfite sequencing data. We demonstrated its superior performance relative to similar methods, and through several case studies showed how it can be used to study loss of imprinting, $\mathrm{X}$-inactivation and methylation heterogeneity in cancer. We expect that DXM will have high applicability and utility for the analysis of subpopulation methylation in any heterogeneous sample and in the future a similar approach could be adapted to the analysis of cell-free DNA.

\section{DATA AVAILABILITY}

DXM is available under the GNU GPL License on GitHub at https://github.com/edwardslab-wustl/dxm or as a Docker image at https://hub.docker.com/repository/ docker/edwardslab/dxm. DXM was written in Python3 and was tested on CentOS7, though it should work on any *nix system. For DLBCL samples, DXM on average used $300 \mathrm{Mb}$ memory and processed a sample in less than two hours, using one CPU.

All bisulfite sequencing data has been made available as a GEO accession GSE130556.

\section{SUPPLEMENTARY DATA}

Supplementary Data are available at NAR Online.

\section{ACKNOWLEDGEMENTS}

We thank Yu Sun for his input into early versions of the deconvolution algorithm. We thank the Genome Technology Access Center for help with genomic sequencing and the Flow Cytometry Core in the Department of Pathology and Immunology for help with cell sorting experiments. We thank the Washington University School of Medicine (WUSM) Lymphoma Banking Program, Dept. of Medicine, Division of Oncology and N. Bartlett for lymphoma biopsies. We thank the Alvin J. Siteman Cancer Center at Washington University School of Medicine and Barnes-Jewish Hospital in St. Louis, MO. and the Institute of Clinical and Translational Sciences (ICTS) at Washington University in St. Louis, for the use of the Tissue Procurement Core, which provided tissue banking service. The 
Siteman Cancer Center is supported in part by an NCI Cancer Center Support Grant \#P30 CA091842 and the ICTS is funded by the National Institutes of Health's NCATS Clinical and Translational Science Award (CTSA) program grant \#UL1 TR002345.

\section{FUNDING}

NIH awards [R21LM012395 to J.R.E., R01GM108811 to J.R.E., F30CA224687 to J.F., T32HG000045 to J.F., T32GM007200-41 to J.F., R01CA188286 to J.E.P., F31CA221012 to J.M.A.]. Funding for open access charge: Institutional Funds.

Conflict of interest statement. None declared.

\section{REFERENCES}

1. Robertson,K.D. (2005) DNA methylation and human disease. Nat. Rev. Genet., 6, 597.

2. McGranahan,N. and Swanton,C. (2017) Clonal heterogeneity and tumor evolution: past, present, and the future. Cell, 168, 613-628.

3. Ding,L., Ley,T.J., Larson,D.E., Miller,C.A., Koboldt,D.C., Welch,J.S., Ritchey,J.K., Young,M.A., Lamprecht,T., McLellan,M.D. et al. (2012) Clonal evolution in relapsed acute myeloid leukaemia revealed by whole-genome sequencing. Nature, 481, 506-510.

4. Landau,D.A., Clement,K., Ziller,M.J., Boyle,P., Fan,J., Gu,H., Stevenson,K., Sougnez,C., Wang,L., Li,S. et al. (2014) Locally disordered methylation forms the basis of intratumor methylome variation in chronic lymphocytic leukemia. Cancer Cell, 26, 813-825.

5. Pan,H., Jiang,Y., Boi,M., Tabbò,F., Redmond,D., Nie,K., Ladetto,M., Chiappella,A., Cerchietti,L., Shaknovich,R. et al. (2015) Epigenomic evolution in diffuse large B-cell lymphomas. Nat. Commun., 6, 6921.

6. Li,S., Garrett-Bakelman,F.E., Chung,S.S., Sanders,M.A., Hricik,T., Rapaport,F., Patel,J., Dillon,R., Vijay,P., Brown,A.L. et al. (2016) Distinct evolution and dynamics of epigenetic and genetic heterogeneity in acute myeloid leukemia. Nat. Med., 22, 792-799.

7. Klughammer,J., Kiesel,B., Roetzer,T., Fortelny,N., Nemc,A., Nenning,K.-H., Furtner,J., Sheffield,N.C., Datlinger,P., Peter,N. et al. (2018) The DNA methylation landscape of glioblastoma disease progression shows extensive heterogeneity in time and space. Nat. Med., 24, 1611-1624.

8. Sheffield,N.C., Pierron,G., Klughammer,J., Datlinger,P., Schönegger,A., Schuster,M., Hadler,J., Surdez,D., Guillemot,D., Lapouble,E. et al. (2017) DNA methylation heterogeneity defines a disease spectrum in Ewing sarcoma. Nat. Med., 23, 386-395.

9. Gaiti,F., Chaligne,R., Gu,H., Brand,R.M., Kothen-Hill,S., Schulman,R.C., Grigorev,K., Risso,D., Kim,K.-T., Pastore,A. et al. (2019) Epigenetic evolution and lineage histories of chronic lymphocytic leukaemia. Nature, 569, 576-580.

10. Beerenwinkel,N., Schwarz,R.F., Gerstung,M. and Markowetz,F. (2015) Cancer Evolution: Mathematical Models and Computational Inference. Syst. Biol., 64, e1-e25.

11. Nautiyal,S., Carlton,V.E.H., Lu,Y., Ireland,J.S., Flaucher,D., Moorhead,M., Gray,J.W., Spellman,P., Mindrinos,M., Berg,P. et al. (2010) High-throughput method for analyzing methylation of CpGs in targeted genomic regions. Proc. Natl. Acad. Sci. U.S.A., 107, 12587-12592.

12. Zhang,W., Spector,T.D., Deloukas,P., Bell,J.T. and Engelhardt,B.E. (2015) Predicting genome-wide DNA methylation using methylation marks, genomic position, and DNA regulatory elements. Genome Biol., 16, 14.

13. Kandoth,C., McLellan,M.D., Vandin,F., Ye,K., Niu,B., Lu,C., Xie,M., Zhang,Q., McMichael,J.F., Wyczalkowski,M.A. et al. (2013) Mutational landscape and significance across 12 major cancer types. Nature, 502, 333-339.

14. Zack,T.I., Schumacher,S.E., Carter,S.L., Cherniack,A.D., Saksena,G., Tabak,B., Lawrence,M.S., Zhang,C.-Z., Wala,J., Mermel,C.H. et al. (2013) Pan-cancer patterns of somatic copy-number alteration. Nat. Genet., 45, 1134-1140.
15. Vidal,E., Sayols,S., Moran,S., Guillaumet-Adkins,A., Schroeder,M.P., Royo,R., Orozco,M., Gut,M., Gut,I., Lopez-Bigas,N. et al. (2017) A DNA methylation map of human cancer at single base-pair resolution. Oncogene, 36, 5648-5657.

16. Titus,A.J., Gallimore,R.M., Salas,L.A. and Christensen,B.C. (2017) Cell-type deconvolution from DNA methylation: a review of recent applications. Hum. Mol. Genet., 26, R216-R224.

17. Chakravarthy,A., Furness,A., Joshi,K., Ghorani,E., Ford,K., Ward,M.J., King,E.V., Lechner,M., Marafioti,T., Quezada,S.A. et al. (2018) Pan-cancer deconvolution of tumour composition using DNA methylation. Nat. Commun., 9, 3220.

18. Rahmani,E., Zaitlen,N., Baran,Y., Eng,C., Hu,D., Galanter,J., Oh,S., Burchard,E.G., Eskin,E., Zou,J. et al. (2017) Correcting for cell-type heterogeneity in DNA methylation: a comprehensive evaluation. Nat. Methods, 14, 218-219.

19. Zheng,S.C., Beck,S., Jaffe,A.E., Koestler,D.C., Hansen,K.D., Houseman,A.E., Irizarry, R.A. and Teschendorff,A.E. (2017) Correcting for cell-type heterogeneity in epigenome-wide association studies: revisiting previous analyses. Nat. Methods, 14, 216-217.

20. Zou,J., Lippert,C., Heckerman,D., Aryee,M. and Listgarten,J. (2014) Epigenome-wide association studies without the need for cell-type composition. Nat. Methods, 11, 309-311.

21. Dedeurwaerder,S., Defrance,M., Bizet,M., Calonne,E., Bontempi,G. and Fuks,F. (2014) A comprehensive overview of Infinium HumanMethylation450 data processing. Brief. Bioinform., 15, 929-941.

22. Zheng,X., Zhao,Q., Wu,H.-J., Li,W., Wang,H., Meyer,C.A., Qin,Q.A., Xu,H., Zang,C., Jiang,P. et al. (2014) MethylPurify: tumor purity deconvolution and differential methylation detection from single tumor DNA methylomes. Genome Biol., 15, 419.

23. Consortium,R.E., Kundaje,A., Meuleman,W., Ernst,J., Bilenky,M., Yen,A., Heravi-Moussavi,A., Kheradpour,P., Zhang,Z., Wang,J. et al. (2015) Integrative analysis of 111 reference human epigenomes. Nature, 518, 317-330.

24. Martens,J.H.A. and Stunnenberg,H.G. (2013) BLUEPRINT: mapping human blood cell epigenomes. Haematologica, $\mathbf{9 8}$, $1487-1489$.

25. The ENCODE Project Consortium (2012) An integrated encyclopedia of DNA elements in the human genome. Nature, $\mathbf{4 8 9}$, $57-74$.

26. Hon,G.C., Hawkins, R.D., Caballero,O.L., Lo,C., Lister,R., Pelizzola,M., Valsesia,A., Ye,Z., Kuan,S., Edsall,L.E. et al. (2012) Global DNA hypomethylation coupled to repressive chromatin domain formation and gene silencing in breast cancer. Genome Res. 22, 246-258.

27. Jiang,Y., Redmond,D., Nie,K., Eng,K.W., Clozel,T., Martin,P., Tan,L.H., Melnick,A.M., Tam,W. and Elemento,O. (2014) Deep sequencing reveals clonal evolution patterns and mutation events associated with relapse in B-cell lymphomas. Genome Biol., 15, 432.

28. Theunissen,T.W., Friedli,M., He,Y., Planet,E., O’Neil,R.C., Markoulaki,S., Pontis,J., Wang,H., Iouranova,A., Imbeault,M. et al. (2016) Molecular criteria for defining the naive human pluripotent state. Cell Stem Cell, 19, 502-515.

29. Schlosberg,C.E., VanderKraats,N.D. and Edwards,J.R. (2017) Modeling complex patterns of differential DNA methylation that associate with gene expression changes. Nucleic Acids Res., 45, $5100-5111$.

30. Feng,H., Conneely,K.N. and Wu,H. (2014) A Bayesian hierarchical model to detect differentially methylated loci from single nucleotide resolution sequencing data. Nucleic Acids Res., 42, e69.

31. O'Leary,N.A., Wright,M.W., Brister,J.R., Ciufo,S., Haddad,D., McVeigh,R., Rajput,B., Robbertse,B., Smith-White,B., Ako-Adjei,D. et al. (2016) Reference sequence (RefSeq) database at NCBI: current status, taxonomic expansion, and functional annotation. Nucleic Acids Res., 44, D733-D745.

32. Schlosberg,C.E., VanderKraats,N.D. and Edwards,J.R. (2017) Modeling complex patterns of differential DNA methylation that associate with gene expression changes. Nucleic Acids Res., 45, $5100-5111$.

33. Court,F., Tayama,C., Romanelli,V., Martin-Trujillo,A., Iglesias-Platas,I., Okamura,K., Sugahara,N., Simón,C., Moore,H., Harness,J.V. et al. (2014) Genome-wide parent-of-origin DNA methylation analysis reveals the intricacies of human imprinting and 
suggests a germline methylation-independent mechanism of establishment. Genome Res., 24, 554-569.

34. Xi,Y. and Li,W. (2009) BSMAP: whole genome bisulfite sequence MAPping program. BMC Bioinformatics, 10, 232.

35. Li,H. (2011) Tabix: fast retrieval of sequence features from generic TAB-delimited files. Bioinformatics, 27, 718-719.

36. Dorri,F., Mendelowitz,L. and Bravo,H.C. (2016) methylFlow: cell-specific methylation pattern reconstruction from high-throughput bisulfite-converted DNA sequencing. Bioinformatics, 32, 1618-1624.

37. Li,L.-C. and Dahiya,R. (2002) MethPrimer: designing primers for methylation PCRs. Bioinformatics, 18, 1427-1431.

38. Tusnády,G.E., Simon,I., Váradi,A. and Arányi,T. (2005) BiSearch: primer-design and search tool for PCR on bisulfite-treated genomes. Nucleic Acids Res., 33, e9.

39. McDonald,J.I., Celik,H., Rois,L.E., Fishberger,G., Fowler,T., Rees,R., Kramer,A., Martens,A., Edwards,J.R. and Challen,G.A (2016) Reprogrammable CRISPR/Cas9-based system for inducing site-specific DNA methylation. Biol. Open, 5, 866-874.

40. Krueger,F. and Andrews,S.R. (2011) Bismark: a flexible aligner and methylation caller for Bisulfite-Seq applications. Bioinforma. Oxf. Engl., 27, 1571-1572.

41. Li,Y. and Sasaki,H. (2011) Genomic imprinting in mammals: its life cycle, molecular mechanisms and reprogramming. Cell Res., 21, 466-473.

42. Schuyler,R.P., Merkel,A., Raineri,E., Altucci,L., Vellenga,E., Martens,J.H.A., Pourfarzad,F., Kuijpers,T.W., Burden,F., Farrow,S. et al. (2016) Distinct trends of DNA methylation patterning in the innate and adaptive immune systems. Cell Rep., 17, 2101-2111.

43. Issa,J.-P. (2014) Aging and epigenetic drift: a vicious cycle. J. Clin. Invest., 124, 24-29.

44. Deaton,A.M. and Bird,A. (2011) CpG islands and the regulation of transcription. Genes Dev, 25, 1010-1022.

45. Duncan,C.G., Grimm,S.A., Morgan,D.L., Bushel,P.R., Bennett,B.D., Roberts,J.D., Tyson,F.L., Merrick,B.A. and Wade,P.A. (2018) Dosage compensation and DNA methylation landscape of the X chromosome in mouse liver. Sci. Rep., 8, 10138.

46. Queirós,A.C., Beekman,R., Vilarrasa-Blasi,R., Duran-Ferrer,M., Clot,G., Merkel,A., Raineri,E., Russiñol,N., Castellano,G., Beà,S. et al. (2016) Decoding the DNA methylome of mantle cell lymphoma in the light of the entire B cell lineage. Cancer Cell, 30, 806-821.

47. Chen,L., Ge,B., Casale,F.P., Vasquez,L., Kwan,T., Garrido-Martín,D., Watt,S., Yan,Y., Kundu,K., Ecker,S. et al. (2016) Genetic drivers of epigenetic and transcriptional variation in human immune cells. Cell, 167, 1398-1414.

48. Gaspar,J.M. and Hart,R.P. (2017) DMRfinder: efficiently identifying differentially methylated regions from MethylC-seq data. $B M C$ Bioinformatics, 18, 528.

49. Bock,C. (2012) Analysing and interpreting DNA methylation data. Nat. Rev. Genet., 13, 705-719.

50. Singer,B.D. (2019) A practical guide to the measurement and analysis of DNA methylation. Am. J. Respir. Cell Mol. Biol., 61, 417-428.

51. Cepek,K.L., Shaw,S.K., Parker,C.M., Russell,G.J., Morrow,J.S., Rimm,D.L. and Brenner,M.B. (1994) Adhesion between epithelial cells and T lymphocytes mediated by E-cadherin and the $\alpha \mathrm{E} \beta 7$ integrin. Nature, 372, 190.

52. Wessel,F., Winderlich,M., Holm,M., Frye,M., Rivera-Galdos,R., Vockel,M., Linnepe,R., Ipe,U., Stadtmann,A., Zarbock,A. et al.
(2014) Leukocyte extravasation and vascular permeability are each controlled in vivo by different tyrosine residues of VE-cadherin. Nat. Immunol., 15, 223-230.

53. Miura,F. and Ito,T. (2015) Highly sensitive targeted methylome sequencing by post-bisulfite adaptor tagging. DNA Res. Int. J. Rapid Publ. Rep. Genes Genomes, 22, 13-18.

54. Ehrlich,M. (2009) DNA hypomethylation in cancer cells. Epigenomics, 1, 239-259.

55. Garrett-Sinha,L.A., Su,G.H., Rao,S., Kabak,S., Hao,Z., Clark,M.R and Simon,M.C. (1999) PU.1 and Spi-B are required for normal B cell receptor-mediated signal transduction. Immunity, 10, 399-408.

56. Tedder,T.F., Tuscano,J., Sato,S. and Kehrl,J.H. (1997) Cd22, a b lymphocyte-specific adhesion molecule that regulates antigen receptor signaling*. Anпи. Rev. Immunol., 15, 481-504.

57. Tessoulin,B., Papin,A., Gomez-Bougie,P., Bellanger,C., Amiot,M., Pellat-Deceunynck,C. and Chiron,D. (2019) BCL2-family dysregulation in B-cell malignancies: from gene expression regulation to a targeted therapy biomarker. Front. Oncol., 8, 645.

58. Spina,V., Khiabanian,H., Messina,M., Monti,S., Cascione,L., Bruscaggin,A., Spaccarotella,E., Holmes,A.B., Arcaini,L., Lucioni,M. et al. (2016) The genetics of nodal marginal zone lymphoma. Blood, 128, 1362-1373.

59. Rasmussen,K.D. and Helin,K. (2016) Role of TET enzymes in DNA methylation, development, and cancer. Genes Dev., 30, 733-750.

60. Spencer,D.H., Russler-Germain,D.A., Ketkar,S., Helton,N.M., Lamprecht,T.L., Fulton,R.S., Fronick,C.C., O'Laughlin,M., Heath,S.E., Shinawi,M. et al. (2017) CpG island hypermethylation mediated by DNMT3A Is a consequence of AML progression. Cell, 168, 801-816.

61. Gnyszka,A., Jastrzębski,Z. and Flis,S. (2013) DNA methyltransferase inhibitors and their emerging role in epigenetic therapy of cancer. Anticancer Res., 33, 2989-2996.

62. Jühling,F., Kretzmer,H., Bernhart,S.H., Otto,C., Stadler,P.F. and Hoffmann,S. (2016) metilene: fast and sensitive calling of differentially methylated regions from bisulfite sequencing data. Genome Res., 26, 256-262.

63. Elliott,G., Hong,C., Xing,X., Zhou,X., Li,D., Coarfa,C., Bell,R.J.A., Maire,C.L., Ligon,K.L., Sigaroudinia,M. et al. (2015) Intermediate DNA methylation is a conserved signature of genome regulation. Nat. Commun., 6, 6363.

64. Tsankov,A.M., Gu,H., Akopian,V., Ziller,M.J., Donaghey,J., Amit,I., Gnirke,A. and Meissner,A. (2015) Transcription factor binding dynamics during human ES cell differentiation. Nature, 518, 344-349.

65. Stadler,M.B., Murr,R., Burger,L., Ivanek,R., Lienert,F., Schöler,A., Nimwegen,E., Wirbelauer,C., Oakeley,E.J., Gaidatzis,D. et al. (2011) DNA-binding factors shape the mouse methylome at distal regulatory regions. Nature, 480, 490-495.

66. Brinkman,A.B., Nik-Zainal,S., Simmer,F., Rodríguez-González,F.G., Smid,M., Alexandrov,L.B., Butler,A., Martin,S., Davies,H., Glodzik,D. et al. (2019) Partially methylated domains are hypervariable in breast cancer and fuel widespread $\mathrm{CpG}$ island hypermethylation. Nat. Commun., 10, 1749.

67. Gaidatzis,D., Burger,L., Murr,R., Lerch,A., Dessus-Babus,S., Schübeler,D. and Stadler,M.B. (2014) DNA sequence explains seemingly disordered methylation levels in partially methylated domains of mammalian genomes. PLOS Genet., 10, e1004143. 